\title{
SRL in der Tasche? - Eine SRL-Interventionsstudie im App-Format
}

\author{
Nora Maria Foerst $\mathbb{D} \cdot$ Andreas Pfaffel · Julia Klug • Christiane Spiel • \\ Barbara Schober
}

Online publiziert: 27. Februar 2019

(C) Der/die Autor(en) 2019

Zusammenfassung Obwohl es bereits einige Studien gibt, die für die Effektivität von App-Interventionen im Bildungskontext sprechen, gibt es bisher keine systematisch evaluierte App zur Förderung von SRL. Im vorliegenden Beitrag wird eine Pilotstudie zu einer SRL-Intervention im App-Format zur Unterstützung von Studierenden beim Schreiben ihrer Bachelorarbeit vorgestellt. Interventionsziel war die Förderung von Wissen zu und Anwendung von metakognitiven SRL-Strategien sowie motivationalen Kompetenzen. In einem quasi-experimentellen Zweigruppenplan $(N=118)$ in den Bachelorarbeit-Seminaren der Studiengänge Psychologie und Wirtschaftswissenschaften, erhielten Studierende der Versuchsgruppe eine SRL-Intervention via Smartphone-App. Die Kontrollgruppe besuchte reguläre Bachelorarbeit-Seminare ohne App-Intervention. Die Wirksamkeit der Intervention wurde mittels PräPost-Fragebogenerhebung sowie ergänzender qualitativer Daten überprüft. Entgegen den Erwartungen zeigte sich im Prä-Post-Vergleich eine Abnahme des selbstberichteten Wissens zu metakognitiven SRL-Strategien. Hinsichtlich deren Anwendung konnten keine signifikanten Veränderungen festgestellt werden. Bei Studierenden, die die App regelmäßig nutzten, konnte zwar eine Steigerung der Motivation zum Schreiben der Bachelorarbeit verzeichnet werden, über die Gruppen hinweg zeigte sich jedoch eine signifikante Zunahme eines ungünstigen Attributionsstils für Erfolg und Misserfolg. Auf Basis dieser Ergebnisse werden Implikationen für eine nach-

Zusatzmaterial online Zusätzliche Informationen sind in der Online-Version dieses Artikels (https:// doi.org/10.1007/s42010-019-00046-7) enthalten.

\footnotetext{
N. M. Foerst $(\varangle) \cdot$ A. Pfaffel · C. Spiel · B. Schober

Fakultät für Psychologie, Institut für Angewandte Psychologie: Arbeit, Bildung Wirtschaft, Universität Wien, Universitätsstraße 7, 1010 Wien, Österreich

E-Mail: nora.foerst@univie.ac.at

J. Klug

Institut für Bildungswissenschaften und Forschung, Pädagogische Hochschule Salzburg,

Akademiestraße 26, 5020 Salzburg, Österreich
} 
haltige Implementierung von SRL-Interventionen sowie den Einsatz von Apps als Interventionstool diskutiert.

Schlüsselwörter Selbstreguliertes Lernen · Metakognition · Motivation · Attribution · Trainingsstudie $\cdot$ Hypermedia

\title{
SRL to go? - Promoting SRL via smartphone-app
}

\begin{abstract}
Despite a number of studies arguing for the effectiveness of app-interventions in the educational context, there are no systematically evaluated apps for promoting SRL, to date. The present pilot study introduces a smartphone-app that aims to support students during the process of their Bachelor's thesis. The intervention focused on the promotion of metacognitive SRL-strategies as well as motivational competences. In a quasi-experimental two-group design $(N=118)$, students of psychology and economic sciences in the bachelor's thesis seminars of the experimental group received an intervention via smartphone-app. The control group attended a regular bachelor's thesis seminar with no app-intervention. In order to evaluate the effectiveness of the intervention, participants answered a prepost-survey and additional qualitative data was collected. Unexpectedly, the prepost-comparison revealed a decline of self-reported knowledge about metacognitive SRL-strategies whereas there was no significant effect for the use of these strategies. Whilst an increase of motivation for writing the bachelor's thesis could be observed in participants that used the app continuously, a significant increase in unfavorable attribution styles for success and failure was found for both groups. Implications for a sustainable implementation of SRL-interventions as well as the eligibility of apps as tools for interventions, are discussed.
\end{abstract}

Keywords Self-regulated learning $\cdot$ Metacognition $\cdot$ Motivation $\cdot$ Attribution · Training $\cdot$ Hypermedia

\section{Einführung}

Beim Übertritt vom schulischen in den universitären Kontext sind junge Erwachsene mit der Herausforderung konfrontiert, sich als autonome Lernende in einem eher unstrukturierten Bildungskontext zurechtzufinden. Studierende sind gefordert, ihren Lernprozess eigenständig zu planen, zu organisieren sowie ihre Lernmotivation und Emotionen zu regulieren. Für diese Anforderungen des selbstregulierten Lernens (SRL) fehlt es jedoch oft an entsprechender Vorbereitung. Obwohl die Fähigkeit zum SRL als Grundlage lebenslangen Lernens gilt (Boekaerts 1997; Wirth und Leutner 2008) und ein explizites Ziel der Hochschulbildung darstellt (European Commission 2008), ist die Befundlage hinsichtlich des Zusammenhangs zwischen SRL und Studienleistung nicht konsistent (Zimmerman 2000). Ein Grund dafür könnte darin bestehen, dass in der Messung von SRL hauptsächlich die deklarative Wissensebene fokussiert wird (vgl. Foerst et al. 2017). Eine weitere Problematik bezüglich der Förderung von SRL besteht darin, dass langfristig wirksame Inter- 
ventionen oft schwer zu implementieren sind. Klassische SRL-Interventionen, in denen in face-to-face Einheiten Wissen vermittelt und SRL trainiert wird, beanspruchen viel Zeit, die innerhalb von Lehrveranstaltungen curricular nicht vorgesehen ist. Daher sollten SRL-Interventionen für den Hochschulkontext möglichst niedrigschwellig und ressourcenschonend gestaltet sein. Neue Medien bieten die Chance, SRL zu fördern, die Nutzung von SRL-Strategien zu erleichtern und dabei einen tieferen Einblick in den Prozess des selbstregulierten Lernens zu erhalten (Azevedo 2005; Dabbagh und Kitsantas 2012; Dignath-van Ewijk et al. 2015). Eine SRL-Intervention via Smartphone-App geht zwar mit einer aufwändigen und kostenintensiven Entwicklung einher, könnte jedoch im Anschluss höchst ökonomisch implementiert werden. Im vorliegenden Artikel wird eine SRL-Intervention im App-Format vorgestellt, die darauf abzielt das Wissen zu sowie die Anwendung von metakognitiven und motivationalen SRL-Strategien bei Studierenden zu fördern.

\section{Theoretischer Hintergrund}

\subsection{SRL im Kontext: Metakognition und Motivation als zentrale Variablen}

SRL umfasst laut Zimmerman (2000) Gedanken, Emotionen und Handlungen sowie deren Planung und zyklische Adaption zur Erreichung der eigenen Ziele. Prozessmodelle des selbstregulierten Lernens (z. B. Schmitz und Wiese 2006; Zimmerman 2000) beinhalten drei aufeinanderfolgende Phasen, die in jeder Lernsituation durchlaufen werden und sich durch charakteristische Aufgaben unterscheiden (vgl. Dignath-van Ewijk et al. 2015; Fabriz et al. 2014; Lan 2005). In der präaktionalen Phase (forethought) stehen Zielsetzung und strategische Planung und im Vordergrund. Hier werden die eigenen Ziele definiert und motivationale Strategien zur Initiierung des Lernprozesses sowie Lernstrategien zur Bewältigung der Aufgabe ausgewählt. Während des eigentlichen Lernvorgangs - der aktionalen Phase (performance) - stehen die Überwachung des eigenen Lernprozesses sowie die Aufrechterhaltung der eigenen Motivation im Vordergrund. Dabei kann sowohl internales (z.B. Vergleich mit dem eigenen Zeitplan) als auch externales Feedback (z. B. Rückmeldung durch Lehrende) genutzt werden. Schließlich werden der eigene Lernprozess sowie seine Resultate in der postaktionalen Phase evaluiert (self-reflection). Lernende nehmen Kausalattributionen ihres Erfolges bzw. Misserfolges vor, die im Sinne eines feedback loops den nachfolgenden Lernprozess beeinflussen.

Metakognitiven SRL-Strategien wird in dem Verständnis von SRL als zyklischen Prozess ein besonderer Stellenwert beigemessen, da diese den Prozess des Lernens selbst regulieren (Dignath-van Ewijk et al. 2015; Schraw et al. 2006). Metakognition umfasst die Planung, Überwachung und Regulation der Vorgehensweise, um die eigenen Ziele zu erreichen (vgl. Mandl und Friedrich 2006). Der Einfluss metakognitiver Strategien auf Lernerfolg und Leistung konnte durch eine Vielzahl von Studien bestätigt werden (vgl. Gomes et al. 2014; Van der Stel und Veenman 2014). Da metakognitive Strategien universell anwendbar und domänenunspezifisch sind, kommt ihnen in wenig strukturierten oder neuen Lernsituationen eine besondere Relevanz zu (Dignath-van Ewijk et al. 2015; Schmidt und Ford 2003). Im Studium 
sind metakognitive Strategien spätestens dann besonders gefordert, wenn es um das Verfassen einer wissenschaftlichen Arbeit geht (Dresel et al. 2015). Von den Studierenden wird erwartet, dass sie ihr Vorgehen über einen langen Zeitraum hinweg eigenständig planen, große Mengen an Literatur aufarbeiten, sich das Schreiben der Arbeit einteilen und immer wieder kontrollieren, ob mit sie mit ihrem bisherigen Vorgehen die Abgabefrist einhalten können.

Allein das Wissen um metakognitive Strategien bedeutet jedoch nicht, dass diese tatsächlich angewandt werden. Um metakognitives Wissen in die Anwendung metakognitiver Strategien zu übersetzen, erfordert es Motivation (Artelt und Wirth 2014; Boekaerts 1999; Rheinberg et al. 2000). Insgesamt spielt Motivation eine essentielle Rolle für den Lernprozess und das Lernergebnis (Richardson et al. 2012; Schmitz 2001; Zimmerman und Schunk 2008). Die kausale Ursachenzuschreibung, die Studierende bezüglich eines Erfolgs bzw. Misserfolgs vornehmen, stellt nicht nur einen Indikator für motivationale Grundmuster dar (Dresel et al. 2005; Pintrich und Schunk 2002), sondern besitzt auch ein hohes Prädiktionspotenzial bezüglich des Umgangs mit zukünftigen Aufgaben (Krapp et al. 2014). Dabei sind insbesondere internale Attribution von Erfolgen sowie variable Attribution von Misserfolgen wichtige Momente eines motivational günstigen Attributionsstils (Dresel 2010). Defensive Schlussfolgerungen bezüglich des Lernergebnisses und ein ungünstiger Attributionsstil können Lernende hingegen daran hindern, das eigene Vorgehen zu adaptieren (Pintrich und Garcia 1994), was zu einer Verschlechterung der Leistung, Gefühlen von Hilflosigkeit, bis hin zum Studienabbruch führen kann (Sageder 1994).

\subsection{SRL-Interventionsansätze - Neue Medien zur Überwindung alter Probleme?}

Zahlreiche Interventionen beschäftigen sich mit der Förderung des selbstregulierten Lernens verschiedener Zielgruppen. Aufgrund der Komplexität und Vielschichtigkeit des Konstrukts SRL fokussieren viele Interventionsansätze auf einzelne Teilbereiche wie kognitive Oberflächen- und Tiefenstrategien (z. B. Rosário et al. 2010). Andere versuchen holistischere Interventionen $\mathrm{zu}$ realisieren, die möglichst viele Facetten von SRL abdecken, um Synergieeffekte von metakognitiven, kognitiven und ressourcenorientierten SRL-Strategien zu nutzen (z. B. Dörrenbächer und Perels 2016a).

Metaanalysen konnten zeigen, dass SRL-Interventionen grundsätzlich positive Effekte auf Lernen, Strategienutzung und Motivation haben können - sogar schon im Grundschulalter (Dignath et al. 2008). Die größten Effekte zeigten sich für motivationale, kognitive und metakognitive Strategien. Am effektivsten waren Interventionen, die kognitive, metakognitive und motivationale Strategien beinhalteten und sowohl Wissen über die Strategien als auch über den Nutzen deren Einsatzes vermittelten (Hattie et al. 1996; Dignath und Büttner 2008).

Die Nützlichkeit von SRL-Interventionen ist damit zwar belegt, in deren Umsetzung und Implementierung entstehen jedoch immer wieder Probleme. Sowohl Interventionen, die auf die Wissensvermittlung von SRL-Teilaspekten fokussieren, als auch breitere Ansätze sind oft mit hohem Aufwand verbunden. Die meisten Interventionsansätze basieren auf face-to-face Einheiten (vgl. Ragosta 2010). Solche 
Interventionen müssen entweder durch die Lehrenden selbst (z. B. Bail et al. 2008) oder durch externe SRL-Trainerinnen und -Trainer durchgeführt werden (z. B. Azevedo und Cromley 2004). Darüber hinaus beanspruchen solche Interventionen Seminarzeit, die curricular nicht vorgesehen ist. Somit scheitert die Umsetzung von SRL-Interventionen trotz nachweisbarer Effektivität häufig an der Implementierung über die Interventionsstudien hinaus (Schmitz und Wiese 2006). Als ein Lösungsansatz für dieses Problem gelten Lerntagebücher, die begleitend zur Intervention und darüber hinaus von den Teilnehmenden bearbeitet werden können (Panadero et al. 2016; Schmitz 2001). Lerntagebücher können zwar die Implementierung und den Transfer einer Intervention fördern, sind jedoch letztlich nicht als Ersatz, sondern als ein Zusatz für SRL-Interventionen zu sehen (Dörrenbächer und Perels 2016b; Fabriz et al. 2014; Schmitz et al. 2011), wodurch die Implementierung weiterhin ressourcenfordernd bleibt. Daher stellt sich die Frage nach weiteren Möglichkeiten zur Erleichterung der Implementierung und Transferförderung von SRL-Interventionen.

Neue Medien bieten grundlegend aussichtsreiche Möglichkeiten, um SRL-Interventionen nachhaltig im Feld zu implementieren. Einige Studien zeigen vielversprechende Ergebnisse für eine virtuelle SRL-Förderung, die ohne Mehraufwand der Lehrenden simultan zum Unterricht am Computer angeboten werden kann (Azevedo und Cromley 2004; Bellhäuser et al. 2016; Hadwin und Winne 2001). Inzwischen sind jedoch Smartphones die meist verbreiteten Mediengeräte (Feierabend et al. 2017). Damit eröffnet sich auch für SRL-Interventionen ein völlig neues Implementierungs-Tool. Während für den Schulbereich bereits einige Smartphone-Apps als Lerninterventionen entwickelt wurden (vgl. Zydney und Warner 2016), sind Studien über Effekte solcher Lerninterventions-Apps im tertiären Bildungsbereich bisher eher begrenzt aussagekräftig. Zwar wurde die generelle Akzeptanz von didaktischen Apps bei Studierenden untersucht (Briz-Ponce und García-Peñalvo 2015; Martin et al. 2013), bisherige App-basierte Interventionen zielen jedoch entweder auf die Vermittlung fachspezifischer Lerninhalte ab (z. B. Briz-Ponce et al. 2016) oder auf die Förderung kollaborativen Lernens, wobei die App lediglich als Gruppen-Diskussionsforum fungiert (z.B. Sung et al. 2017). Bis dato existiert nach Wissen der Autorinnen und Autoren keine SRL-Intervention im App-Format für Studierende.

\subsection{Ziel, Ansatz und Forschungsfragen der Interventionsstudie}

\subsubsection{Interventionsziel}

Hauptziel der vorliegenden Studie war es, eine SRL-Intervention zu entwickeln, pilotieren und evaluieren, die die beschriebenen Probleme von SRL-Interventionsstudien im Hochschulsektor umgehen kann. So stand zum einen nicht die reine Wissensvermittlung, sondern vor allem die Förderung der Anwendung von SRLStrategien im Vordergrund. Zum anderen sollte die Intervention ökonomisch und niedrigschwellig sein, um langfristig und breitenwirksam implementiert werden zu können. 


\subsubsection{Interventionsansatz}

Zur Verfolgung dieses Ziels wurde die Smartphone-App „META“ entwickelt, in Bachelorarbeit-Seminaren implementiert und explorativ evaluiert. In der Intervention werden inhaltlich und didaktisch Elemente etablierter SRL-Interventionen realisiert, die sich als besonders effektiv erwiesen haben. Inhaltlich wurde daher die Förderung von Wissen zu und Anwendung von metakognitiven und motivationalen Strategien - hier insbesondere die für den Umgang mit Misserfolg besonders wichtigen Attributionen - gewählt (vgl. z. B. Artelt und Wirth 2014; Dresel 2010; Gomes et al. 2014; Van der Stel und Veenman 2014). Didaktisch wurden drei Hauptelemente umgesetzt, deren Relevanz im Folgenden kurz dargestellt wird: Zeitmanagement, Lerntagebücher sowie Feedback an die Studierenden.

Ein Hauptelement der Intervention war die Förderung effektiven Zeitmanagements beim Verfassen der Bachelorarbeit. Zeitmanagement spannt als ein Kernelement metakognitiver SRL-Strategien nicht nur den Rahmen, in dem sich alle weiteren Arbeitsschritte vollziehen, sondern dient auch als Grundlage zur Überprüfung, inwieweit das eigene Vorgehen zielführend ist oder gegebenenfalls angepasst werden muss (Macan 1994; Wagner et al. 2006).

Zeitmanagement beinhaltet das Setzen und Hierarchisieren von Zielen sowie die Ableitung von konkreten Aufgaben, die dann geplant und ausgeführt werden müssen (Britton und Glynn 1989). Da die Qualität des Zeitmanagements nachweisbar einen positiven Einfluss auf die akademische Leistung sowie auf motivationale Faktoren ausübt (García-Ros et al. 2004; Klein et al. 2003), gibt es unterschiedlichste Ansätze zur Verbesserung des eigenen Zeitmanagements. Metzger (2002) identifizierte folgende zentrale Elemente effektiver Zeitmanagementtrainings: (a) Effektive Zeiteinteilung (Setzen von Zwischenzielen und Milestones, genügend Zeit einplanen), (b) Zeit gewinnen (z. B. durch Priorisierung von Aufgaben und Zielen), (c) Erstellung eines Zeitplans (zur Visualisierung des Zeitbudgets), (d) Vermeiden von Prokrastination (z. B. durch Selbstbelohnung) und (e) Zeitanalysen (z. B. durch Führen eines Lern-/Arbeitstagebuchs).

Das zweite Hauptelement der Intervention war ein mobiles Lerntagebuch. Lerntagebücher erwiesen sich in der SRL-Forschung sowohl als Messinstrumente, als auch als Interventionselemente als effektiv (Panadero et al. 2016). Da der Transfer einer Intervention in die Praxis durch den Einsatz von Lerntagebüchern sowohl gefördert wird als auch durch die Daten, die Lerntagebücher liefern, nachvollzogen werden kann, sind SRL-Interventionen in Kombination mit einem Lerntagebuch reinen SRL-Interventionen überlegen (Dörrenbächer und Perels 2016b; Schmitz et al. 2011). Darüber hinaus regt die schriftliche Aufzeichnung des Lern- bzw. Arbeitsprozesses metakognitive Überwachungsprozesse an. Die Sichtbarmachung auch kleiner Erfolge und Fähigkeitszuwächse fördert zusätzlich die Motivation von Studierenden (Schmitz et al. 2011).

Als drittes Hauptelement der Intervention wurde Feedback realisiert, auf dessen Basis Studierende ihr eigenes Vorgehen reflektieren und regulieren können. Butler und Winne (1995) plädieren für die Integration von prozessbezogenem Feedback in jegliche SRL-Forschungs- und -Interventionsstudien. Sie postulieren fünf Funktionen, die Feedback im SRL-Prozess erfüllen kann: Sollten Vorhaben und tat- 
sächliches Vorankommen übereinstimmen, kann Feedback (a) als Bestätigung des eigenen Vorgehens dienen und (b) dabei helfen, dieses noch besser auf die Aufgabe abzustimmen. Feedback kann jedoch auch (c) zusätzliche Informationen liefern bzw. (d) falsche Informationen oder Annahmen korrigieren. Bei falschen Annahmen bezüglich der Umsetzung der eigenen Lern- bzw. Arbeitsziele, kann Feedback schließlich dazu dienen, (e) das eigene Vorgehen grundsätzlich neu zu strukturieren. Insgesamt hat prozessbezogenes Feedback einen positiven Einfluss auf Selbstwirksamkeit und Motivation (Carver und Scheier 2000; Schunk und Ertmer 2000).

\subsubsection{Forschungsfragen}

Da ein besonderes Augenmerk der Intervention darauf liegt, nicht nur das Wissen $\mathrm{zu}$, sondern auch die Anwendung von Strategien zu fördern und die Intervention inhaltlich auf metakognitive und motivationale Strategien fokussiert, lauten die ersten beiden Forschungsfragen wie folgt:

1. Fördert die Nutzung der SRL-Interventions-App „META“ sowohl das Wissen zu als auch die Anwendung von metakognitiven Strategien?

2. Fördert die Nutzung der SRL-Interventions-App „META“ motivationale Variablen im Umgang mit der Bachelorarbeit (Motivation zum Schreiben der Bachelorarbeit, Attributionsstil)?

Um die Relevanz der Intervention zu untermauern und aufgrund der widersprüchlichen Befundlage zum Zusammenhang zwischen SRL und Studienleistung (Zimmerman 2000), lautet die dritte Forschungsfrage:

3. Verbessert die Nutzung der SRL-Interventions-App „META“ die Studienleistung (Notendurchschnitt der letzten drei Vorlesungs-Prüfungen, Note der letzten kleinen wissenschaftlichen Arbeit, Note der Bachelorarbeit)?

Um zu überprüfen, ob die Intervention von den Studierenden überhaupt wie erwünscht implementiert und genutzt wurde, dient die vierte Forschungsfrage als Manipulation-Check:

4. Wird die SRL-Interventions-App „META“ von den Studierenden als nützlich empfunden und genutzt?

\section{Methode}

\subsection{Design und Durchführung der Studie}

Als Design der Interventionsstudie wurde ein Prä-Post-Zweigruppenplan (Oktober 2016 und Januar 2017) vorgesehen. Die Zielgruppe der Intervention waren Studierende, die ein Bachelorarbeit-Seminar besuchten. Ziel der Bachelorarbeit-Seminare, die am Ende des Bachelorstudiums belegt werden, ist die Vermittlung der Regeln guter wissenschaftlicher Praxis und kritischer Auseinandersetzung mit einem Thema, zu dem im Rahmen des Seminars eine schriftliche Bachelorarbeit erstellt werden 
soll. Jene Studierenden, die der Versuchsgruppe (VG) zugeteilt wurden, nahmen an einem Bachelorarbeit-Seminar teil, erhielten Zugang zur App, Instruktionen zur App-Nutzung sowie den App-Inhalten und nahmen an zwei Reflexionseinheiten zur App teil. Die Nutzung der App im Laufe des Semesters war jedoch freiwillig. Jene Studierenden, die der Kontrollgruppe (KG) zugeteilt wurden, nahmen nur an einem Bachelorarbeit-Seminar teil und hatten keine Möglichkeit zur Nutzung der App. Für einen detaillierten Überblick des Ablaufs der Intervention siehe Tab. 1.

\subsection{Stichprobe}

Zur Rekrutierung wurden die Lehrveranstaltungsleiterinnen und -leiter aller Bachelorarbeit-Seminare im Wintersemester 2016/17 in den Studienrichtungen Psychologie und Wirtschaftswissenschaften der Universität Wien kontaktiert. Von den insgesamt 13 Bachelorarbeit-Seminaren konnten sechs Seminare (46\%) für die Teilnahme an der Studie gewonnen werden. Die Stichprobe bestand aus insgesamt $N=118$ Studierenden der Universität Wien (79 Frauen, 33 Männer, 6 Personen ohne Angabe des Geschlechts), wobei 79 Personen aus dem Studiengang Psychologie und 39 Personen

Tab. 1 Ablaufplan der Intervention

\begin{tabular}{|c|c|c|c|c|}
\hline Zeitpunkt & Art der Erhebung & Inhalte & Min. & $N$ \\
\hline \multirow[t]{9}{*}{$\overline{10 / 2016}$} & \multirow{9}{*}{$\begin{array}{l}\text { Paper-Pencil; } \\
\text { Quantitativ }\end{array}$} & Prätest & $\overline{45}$ & $118(\mathrm{VG}$, \\
\hline & & $\begin{array}{l}\text { Sub-Fragebogen „Metakognition“ des } \\
\text { SRL-QuAK }\end{array}$ & & \\
\hline & & $\begin{array}{l}\text { Items zur Attribution von Erfolg/ } \\
\text { Misserfolg }\end{array}$ & & \\
\hline & & $\begin{array}{l}\text { Notendurchschnitt der letzten drei Vorle- } \\
\text { sungs-Prüfungen }\end{array}$ & & \\
\hline & & $\begin{array}{l}\text { Note der letzten kleinen wissenschaftli- } \\
\text { chen Arbeit }\end{array}$ & & \\
\hline & & App-Instruktion & 45 & $69(\mathrm{VG})$ \\
\hline & & Vorstellung des Projektes & 5 & \\
\hline & & Vorstellung der App & 20 & \\
\hline & & Instruktion zur App & 20 & \\
\hline \multirow[t]{9}{*}{$11 / 2016$} & \multirow{9}{*}{$\begin{array}{l}\text { Offene } \\
\text { Diskussion; } \\
\text { Qualitativ }\end{array}$} & Reflexion & 60 & $69(\mathrm{VG})$ \\
\hline & & Einstellung zur App & & \\
\hline & & Häufigkeit der App-Nutzung & & \\
\hline & & $\begin{array}{l}\text { Nützlichkeitseinschätzung der einzelnen } \\
\text { Funktionen }\end{array}$ & & \\
\hline & & $\begin{array}{l}\text { Auswirkungen der App auf das eigene } \\
\text { Arbeitsverhalten }\end{array}$ & & \\
\hline & & Motivation zur weiteren App-Nutzung & & \\
\hline & & Positive/negative Aspekte der App & & \\
\hline & & $\begin{array}{l}\text { Umsetzung der in der App behandelten } \\
\text { Strategien }\end{array}$ & & \\
\hline & & $\begin{array}{l}\text { Probleme/Lösungen hinsichtlich der } \\
\text { App-Nutzung/des Schreibens der Bache- } \\
\text { lorarbeit }\end{array}$ & & \\
\hline
\end{tabular}


Tab. 1 (Fortsetzung)

\begin{tabular}{|c|c|c|c|c|}
\hline Zeitpunkt & Art der Erhebung & Inhalte & Min. & $N$ \\
\hline \multirow[t]{8}{*}{$\overline{01 / 2017}$} & \multirow{5}{*}{$\begin{array}{l}\text { Paper-Pencil; } \\
\text { Quantitativ }\end{array}$} & Posttest & \multirow[t]{5}{*}{45} & \multirow{5}{*}{$\begin{array}{l}\mathbf{1 1 8}(\mathrm{VG}, \\
\mathrm{KG})\end{array}$} \\
\hline & & $\begin{array}{l}\text { Sub-Fragebogen „Metakognition“ des } \\
\text { SRL-QuAK }\end{array}$ & & \\
\hline & & $\begin{array}{l}\text { Items zur Attribution von Erfolg/ } \\
\text { Misserfolg }\end{array}$ & & \\
\hline & & $\begin{array}{l}\text { Notendurchschnitt der letzten drei Vorle- } \\
\text { sungs-Prüfungen }\end{array}$ & & \\
\hline & & $\begin{array}{l}\text { Note der letzten kleinen wissenschaftli- } \\
\text { chen Arbeit }\end{array}$ & & \\
\hline & \multirow{3}{*}{$\begin{array}{l}\text { Offene } \\
\text { Diskussion, } \\
\text { Qualitativ }\end{array}$} & Reflexion & \multirow[t]{3}{*}{15} & \multirow[t]{3}{*}{$69(\mathrm{VG})$} \\
\hline & & Feedback zur Intervention & & \\
\hline & & Reflexion zur eigenen Nutzung & & \\
\hline \multirow{12}{*}{$\begin{array}{l}03 / \text { bis } \\
05 / 2017\end{array}$} & \multirow{6}{*}{$\begin{array}{l}\text { Online } \\
\text { Quantitativ }\end{array}$} & Follow-up & \multirow[t]{12}{*}{10} & \multirow{12}{*}{$\begin{array}{l}46(\mathrm{VG}, \\
\mathrm{KG})\end{array}$} \\
\hline & & Bachelorarbeit/Bachelorarbeit-Seminar & & \\
\hline & & Note Bachelorarbeit(/-Seminar) & & \\
\hline & & $\begin{array}{l}\text { Subjektive Relevanz der eigenen Leis- } \\
\text { tung bzgl. Bachelorarbeit(//Seminar) }\end{array}$ & & \\
\hline & & $\begin{array}{l}\text { Erfolgserleben hinsichtlich Bachelorar- } \\
\text { beit(/-Seminar) }\end{array}$ & & \\
\hline & & $\begin{array}{l}\text { Items zur Attribution von Erfolg/ } \\
\text { Misserfolg }\end{array}$ & & \\
\hline & \multirow[t]{3}{*}{ Quantitativ } & Smartphone-App „META“ & & \\
\hline & & Nutzungshäufigkeit der App & & \\
\hline & & $\begin{array}{l}\text { Nützlichkeitseinschätzung der einzelnen } \\
\text { Funktionen und der App insgesamt }\end{array}$ & & \\
\hline & \multirow[t]{3}{*}{ Qualitativ } & Gründe für seltene Nutzung der App & & \\
\hline & & Optimierungsfeedback & & \\
\hline & & $\begin{array}{l}\text { Einstellung zu Apps als Unterstützung } \\
\text { bei einer Bachelorarbeit/im Studium } \\
\text { generell }\end{array}$ & & \\
\hline
\end{tabular}

Min. Minuten, $N$ Stichprobengröße, $V G$ Versuchsgruppe, $K G$ Kontrollgruppe

aus den Wirtschaftswissenschaften stammten. Die Studierenden waren zwischen 20 und 42 Jahre alt $(M=23,5, S D=2,64)$ und studierten im 4 . bis 15 . Semester $(M=7,5$, $S D=1,77)$. Zwischen den Studienrichtungen gab es keine signifikanten Unterschiede bezüglich Geschlecht $\left(\chi^{2}(1)=3,393, p=0,065\right)$ und Alter $(F(1,2370,7)=0,933$, $p=0,334$ ).

Die VG umfasste insgesamt 63, die KG 55 Studierende. Zwischen VG und KG zeigten sich zum ersten Messzeitpunkt keine Unterschiede bezüglich Geschlecht $\left(\chi^{2}(1)=0,018, p=0,894\right)$, Alter $(t(116)=1,517, p=0,132)$ und Semesteranzahl $(t(116)=1,639, p=0,104)$. Auch zeigten sich keine signifikanten Unterschiede bezüglich der Verteilung der beiden Studienrichtungen $\left(\chi^{2}(1)=0,214, p=0,644\right)$ auf VG (41 Psychologie, 22 Wirtschaftswissenschaften) und KG (38 Psychologie, 17 Wirtschaftswissenschaften). 
An der Follow-up-Erhebung nahmen 26 Studierende der VG sowie 20 Studierende der KG teil.

\subsection{Erhebungsinstrumente}

\subsubsection{Prä- und Posttest}

Zur Erfassung von Wissen zu und Anwendung von metakognitiven SRL-Strategien wurde der entsprechende Sub-Fragebogen des SRL-QuAK (Self-Regulated Learning: Questionnaire for Action and Knowledge; Foerst et al. 2017) eingesetzt. Zu Beginn des Fragebogens wurde eine Vignette vorgegeben, in der die Studierenden gebeten wurden, sich in die Situation des Erstellens einer kleinen wissenschaftlichen Arbeit (z. B. Seminararbeit, Bachelorarbeit oder Essay) hineinzuversetzen. Es folgte eine kurze Beschreibung der formalen Herausforderungen der Situation (Literatur eigenständig aufarbeiten, eigenständige Fertigstellung der Arbeit zu einem gegebenen Zeitpunkt) sowie die Information, dass viele Studierende angaben, dass es in dieser Situation günstig sei, über das eigene Vorgehen nachzudenken. Im Anschluss folgten Items zu metakognitiven SRL-Strategien (z. B. ,Ich erstelle mir einen Zeitplan, bei dem ich nicht nur die wissenschaftliche Arbeit berücksichtige, sondern auch andere Tätigkeiten und Zeit für Erholung einplane."). Für jedes Item sollten die Teilnehmenden auf einer visuellen Analogskala durch Ankreuzen der entsprechenden Stelle angeben, für wie günstig sie diese Strategie halten (,,sehr ungünstig“ bis „sehr günstig“) und wie häufig sie diese Strategie tatsächlich anwenden („nie“ bis ,,immer"). Die Markierungen der Studierenden wurden mittels AuswertungsSchablonen den numerischen Werten (1 bis 10) der Analogskala zugeordnet und in SPSS übertragen. Die Reliabilitäten der Skalen sind mit einem Cronbachs Alpha von 0,93 für die Skala „Wissen zu metakognitiven Strategien“ und einem Cronbachs Alpha von 0,79 für die Skala „Anwendung von metakognitiven Strategien“ als gut zu bewerten.

Es folgten Einzelitems zur Attribution (vgl. Dresel et al. 2005) bei Erfolg (z.B. „Wenn ich bei einer kleinen wissenschaftlichen Arbeit einen Erfolg habe, liegt das hauptsächlich daran, dass ich mich angestrengt habe.“) und Misserfolg (z. B. ,Wenn ich bei einer kleinen wissenschaftlichen Arbeit einen Misserfolg habe, liegt das hauptsächlich daran, dass ich Pech hatte.“). Entsprechend des Vierfelderschemas der Attribution nach Weiner (1985) mit den zwei Dimensionen Lokation (interne vs. externe Ursachen) und Stabilität (stabile vs. variable Ursachen) wurden für Erfolgs- und Misserfolgssituationen je vier Aussagen vorgegeben. Die Zustimmung der Studierenden zu den insgesamt acht Aussagen wurde auf einer 10-stufigen Skala

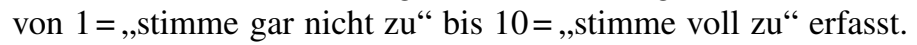

Objektive Leistungskriterien wurden anhand (a) der Note auf die letzte kleine wissenschaftliche Arbeit sowie (b) der letzten drei schriftlichen Vorlesungs-Prüfungsnoten erhoben. Die Bearbeitung dauerte in etwa 45 Minuten und erfolgte im Hörsaal im Paper-Pencil Format. 


\subsubsection{Reflexionseinheiten}

Um Aufschluss über die Rezeption und Nutzung der App zu erhalten, wurden in den Seminaren der VG jeweils zwei face-to-face Reflexionseinheiten durchgeführt und schriftlich mitprotokolliert. Die Reflexionseinheiten zielten darauf ab, Feedback seitens der Studierenden zur App einzuholen sowie den Studierenden einen Anstoß zur weiteren Nutzung der App zu geben. Die im offenen Diskussionsformat behandelten Fragen sind Tab. 1 zu entnehmen.

\subsubsection{Follow-up-Erhebung}

Die Follow-up-Erhebung erfolgte mittels Online-Fragebogen nach dem Abschluss der Benotung des jeweiligen Bachelorarbeit-Seminars. Den Studierenden der VG und KG wurde per Mail der Link zur online Umfrage via unipark (Questback GmbH 2016) zugesandt. Die Bearbeitungsdauer betrug in etwa 10 Minuten. Erfragt wurden die Note der Bachelorarbeit, die Note des Bachelorarbeit-Seminars sowie die Ursachenzuschreibung über die Leistung in der Bachelorarbeit. Die Teilnehmenden gaben an, wie wichtig ihnen eine gute Leistung bezüglich der Bachelorarbeit und des Bachelorarbeit-Seminars war. Die VG beantwortete zusätzlich Fragen zur Nutzung und Nützlichkeit der App, ihrer einzelnen Funktionen sowie zu Gründen für eine seltene Nutzung der App. Das Antwortformat des quantitativen Teils (vgl. Tab. 1) stellte sich als 10-stufige Skala mit den Polen $1=$,stimme gar nicht zu“ bis $10=$,stimme voll zu“ dar, die qualitativen Daten (vgl. Tab. 1) wurden im offenem Antwortformat erfasst.

\subsubsection{Log-files}

Auf Basis der Log-Files wurden Informationen über den Zeitraum der Nutzung der App über das Semester hinweg (Beginn der Intervention bis zum letzten Login) sowie über die Nutzungsintensität (Anzahl der Klicks) der Studierenden gesammelt.

\subsection{Smartphone-App „META“}

Die App „META“ wurde in Kooperation mit der Fakultät für Computerwissenschaft (Research Group Entertainment Computing) der Universität Wien entwickelt. Im Folgenden werden die vier Grundfunktionen der App - Module, Zeitplan, Tagebuch und Feedback - kurz vorgestellt. Jede der Grundfunktionen umfasst sowohl Komponenten in denen Wissen über den Nutzen und die Anwendbarkeit der vorgestellten SRL-Strategien vermittelt wird, als auch Komponenten zur direkten interaktiven Umsetzung der jeweiligen Strategien.

\subsubsection{Module}

Über die Funktion Module (Abb. 1) wurde den Studierenden das notwendige Wissen vermittelt, um die vorgestellten SRL-Strategien auf eine konkrete Aufgabe anzuwenden. Die Module Planung, Überwachung und Regulation waren einmalig durchzu- 
a

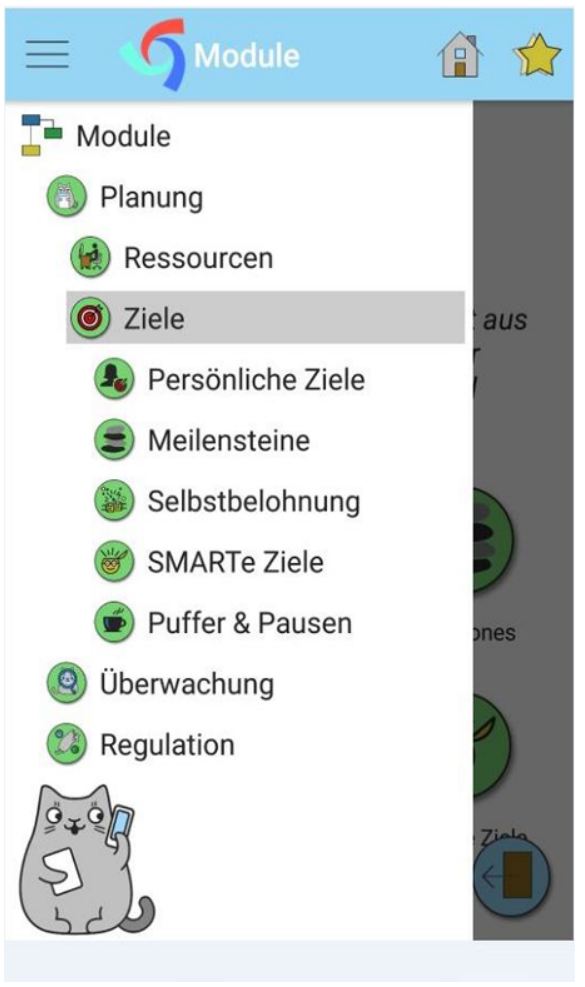

b

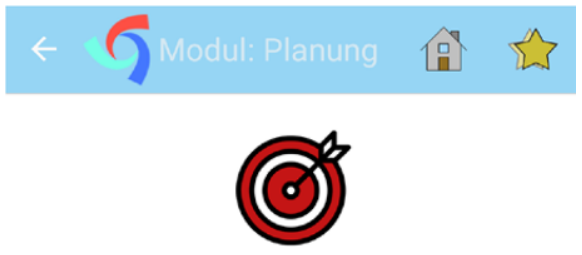

"Der Langsamste, der sein Ziel nicht aus den Augen verliert, geht noch immer geschwinder, als jener, der ohne Ziel umherirrt" - Lessing

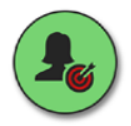

Persönliche Ziele

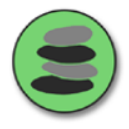

Milestones
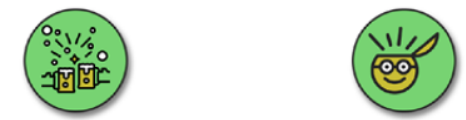

SMARTe Ziele
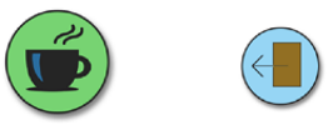

Abb. 1 Smartphone-App „META“ Screenshots: a Reiter, b Modul Planung - Unterpunkte „Ziele“

arbeiten, während die weiteren Funktionen der App (Zeitplan, Tagebuch und Feedback) kontinuierlich über das Semester hinweg genutzt werden sollten. Das Modul Planung teilte sich in Ressourcenplanung (intern und extern) und die Planung von Zielen. Die Zielplanung umfasste die Angabe der persönlichen Ziele für die Bachelorarbeit, die Auflistung antizipierter Milestones (mit anschließender Übertragung in den Zeitplan), die Angabe von Selbstbelohnungsmöglichkeiten bei der Erreichung eines Milestones, Input zur Relevanz der Berücksichtigung von Puffer \& Pausen sowie video- und grafikbasierter Input zur Entwicklung „SMART“-er Ziele (Conzemius und O'Neill 2009). Die Studierenden hatten jederzeit die Möglichkeit ihre Einträge wieder aufzurufen und gegebenenfalls zu adaptieren. In den beiden restlichen Modulen wurde nach einem kurzen theoretischen Input zu den beiden Funktionen Zeitplan und Tagebuch übergeleitet, die konstant über das Semester hinweg zur Überwachung und Regulation des Schreibprozesses der Bachelorarbeit genutzt werden sollten. In der Vorstellung des Zeitplans wurde explizit dazu ermutigt, diesen über das Semester hinweg immer wieder zu prüfen und gegebenenfalls anzupassen. 


\title{
3.4.2 Zeitplan
}

Der Zeitplan stellte die Basis für ein effektives Zeitmanagement dar. In dieser klassischen Kalenderfunktion konnten manuell Termine eingefügt sowie die Milestones aus dem Planungsmodul per drag \& drop übernommen und jederzeit verschoben werden. Die App erinnerte via push-Nachrichten an bevorstehende Milestones und fragte am definierten Fälligkeitsdatum, ob diese erreicht wurden.

\subsubsection{Tagebuchfunktion}

Die Tagebuchfunktion gliederte sich in (a) My Diary, (b) EMoTracker, (c) Strategy Tracker sowie (d) Effizienzabfrage. My Diary war ein Lerntagebuch mit offenen Fragen zur kontinuierlichen, selbstständigen Erfassung des Schreibprozesses (vgl. Landmann und Schmitz 2007). Die Funktion sollte es den Studierenden ermöglichen, ihren Schreibprozess zu dokumentieren, zu überprüfen und dann auf Basis der Aufzeichnungen gegebenenfalls zu adaptieren. Im EMoTracker (Abb. 2a) waren die eigene Motivation am jeweiligen Tag an der Bachelorarbeit zu arbeiten, die aktuelle Zufriedenheit mit der eigenen Leistung, die Konzentrationsfähigkeit und das Durchhaltevermögen in Bezug auf das Schreiben der Bachelorarbeit sowie die allge-

a

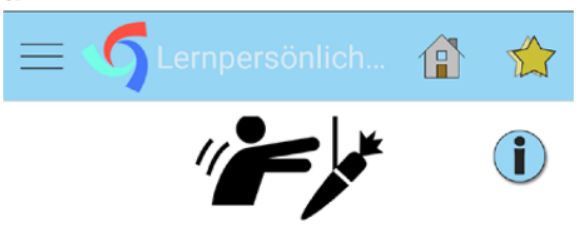

Wie motiviert bist du heute, an deiner BA zu schreiben?

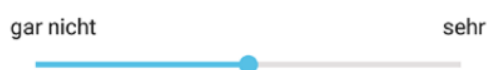

\section{Zufriedenheit}

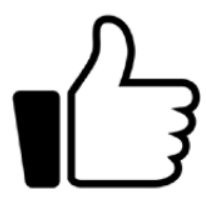

Wie zufrieden bist du heute mit deiner eigenen Leistung beim Schreiben der BA? b

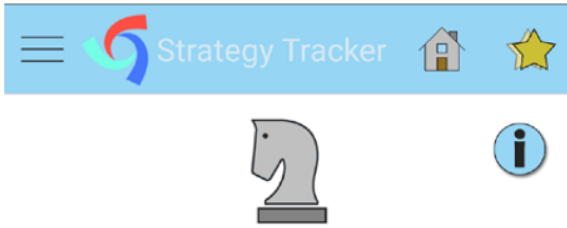

Wie gut ist es dir heute gelungen, zu planen, welche Ressourcen du benötigst?

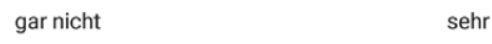

Wie gut ist es dir heute gelungen, deine Ziele zu erreichen?

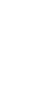


meine Stimmung einzuschätzen. Im Strategy Tracker (Abb. 2b) war anzugeben, wie gut es am jeweiligen Tag gelungen war, die in der Intervention fokussierten SRLStrategien umzusetzen. Diese Strategien umfassten die Ressourcenplanung, die Zielerreichung, die Einhaltung von Pausen, das Setzen von Milestones und SMART-en Zielen, die Selbstbelohnung, die Überwachung sowie Reflektion und Regulation des Schreibprozesses. Die Beantwortung der Fragen der beiden Tracker erfolgte via Schieberegler mit den Polen 0 ,gar nicht“ bis 4 ,sehr“/,sehr gut“. Die Effizienzabfrage erfolgte automatisch beim Öffnen der App. Die Studierenden wurden gebeten anzugeben, wieviel Zeit sie heute für die Bachelorarbeit aufwenden möchten. Beim nächsten Öffnen der App war anzugeben, ob die von ihnen geplante Zeit tatsächlich für die Bachelorarbeit aufgewandt wurde.

\subsubsection{Feedback}

Das Ausfüllen der beiden Tracker sowie die Effizienzabfrage stellten den Datenpool für das graphisch als Verlaufsdiagramme dargestellte Feedback. Hier konnten Verlaufsdiagramme bezüglich des EMo- und Strategy Trackers als auch der eigenen Effizienz (Abb. 3) eingesehen werden. Für die Bearbeitung der App (Durcharbeiten

a

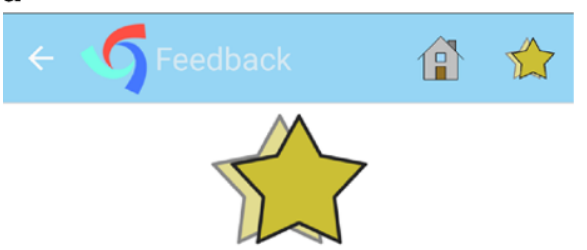

Level 2
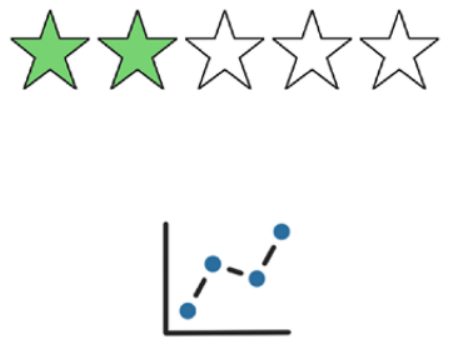

\section{Diagramme}
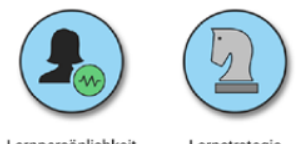

Lernstrategie

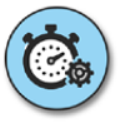

Effizienz b

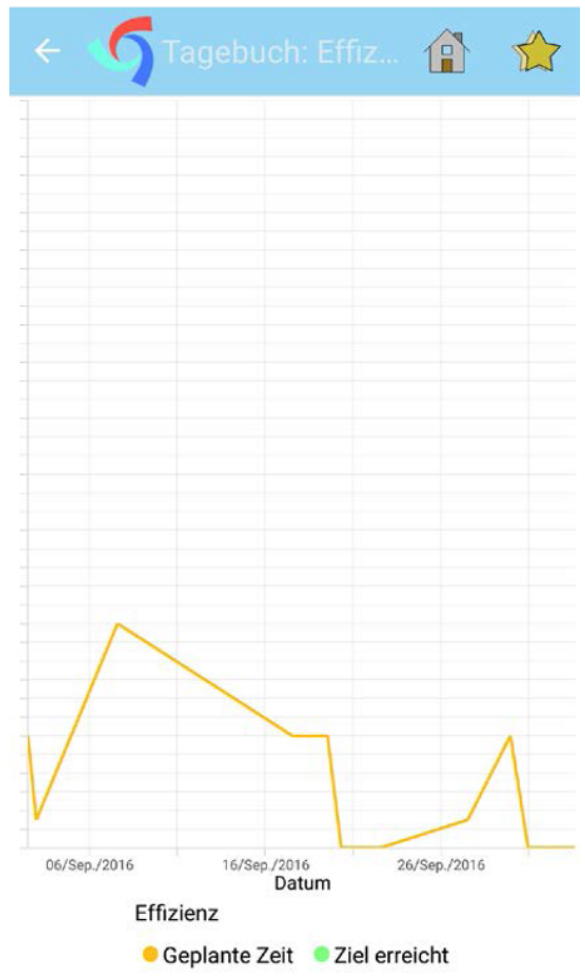

Abb. 3 Smartphone-App „META“ Screenshots: a Feedback - Übersichtsseite, b Feedback - Effizienz Verlaufssdiagramm 
der Module, Erreichen von Milestones etc.) erhielten die Teilnehmenden Aktionspunkte, die nach Erreichen einer bestimmten Anzahl zum Aufstieg in das nächste Level führten. Für das Erreichen des ersten Levels waren 1000 Aktionspunkte nötig, mit 40.000 Aktionspunkten erreichte man das fünfte und höchste Level.

\subsection{Analyse der Daten}

\subsubsection{Analyse der quantitativen Daten}

Zur Prüfung der Interventionseffekte wurden $2 \times 2$-faktorielle Kovarianzanalysen mit Messwiederholung gerechnet, mit dem Faktor Untersuchungsgruppe (VG, KG), der Kontrollvariable Studienrichtung sowie den Kovariaten Nutzungsintensität und Zeitraum der Nutzung der App über das Semester hinweg (vgl. Zusätzliches Onlinematerial A). Die abhängigen Variablen waren: 1) Wissen zu und Anwendung von metakognitiven Strategien, 2) Attribution von Erfolg bzw. Misserfolg sowie 3) die Leistungsindikatoren Durchschnittsnote der letzten drei absolvierten VorlesungsPrüfungen und die Note auf die letzte kleine wissenschaftliche Arbeit. Die Evaluationsstudie war so angelegt, dass ein Interaktionseffekt von mindestens $5 \%$ erklärter Varianz in den Kovarianzanalysen mit Messwiederholung aufgedeckt werden konnte. Bei einer Stichprobe von $N=118$, einer angenommen maximalen Irrtumswahrscheinlichkeit von $\alpha=0,05$, einer angenommenen Korrelation der Messwiederholung von 0,30 und einer erwarteten Effektgröße der Interaktion von $\eta_{p}^{2} \geq 0,05$, beträgt die Teststärke $(1-\beta)$ ca. 98,7\% (berechnet mit $G^{*}$ Power, Version 3.1.9.2).

Zur Prüfung von Veränderungen der Motivation zum Schreiben der Bachelorarbeit über die Zeit hinweg wurden die Angaben zur Motivation zum Schreiben an der Bachelorarbeit am jeweiligen Tag aus dem EMoTracker herangezogen. Diese wurden anhand eines lineares Wachstumsmodells mit Zeit als Kovariate mit festen und zufälligen Effekten modelliert.

\subsubsection{Umgang mit fehlenden Werten}

Von insgesamt 46 Fällen (39\%) lagen die Daten nur zu einem Messzeitpunkt vor. Konkret waren dies zum Prätest fünf Fälle $(4,2 \%)(\mathrm{VG}=2, \mathrm{KG}=3)$ und zum Posttest 41 Fälle $(34,7 \%)(\mathrm{VG}=22, \mathrm{KG}=19) . \mathrm{Zu}$ beiden Messzeitpunkten machten jeweils fünf Personen keine Angaben zu einzelnen Items, wobei der Anteil an nicht beantworteten Items insgesamt unter $5 \%$ lag.

Die fehlenden Werte zu den Messzeitpunkten sowie einzelne fehlende Werte wurden mittels multivariater Imputation mit Markovketten mit dem mice-package (vgl. van Buuren und Groothuis-Oudshoorn 2011, Version 2.46.0) in R-Statistics geschätzt (s. Zusätzliches Onlinematerial B).

\subsubsection{Analyse der qualitativen Daten}

Um einen differenzierteren Einblick in die Rezeption der Intervention und das Nutzungsverhalten der Studierenden zu erhalten wurden in der Interventionsstudie auch qualitative Daten erhoben (siehe Tab. 1). In einem ersten Schritt wurde eine induk- 
tive Kategorisierung der Beiträge aus den Reflexionseinheiten vorgenommen. Auf Basis der so gewonnenen Kategorien wurden die offenen Antworten aus der Followup-Erhebung durch zwei wissenschaftliche Mitarbeiterinnen kategorisiert. Die Interrater-Übereinstimmung für die Kategorisierung betrug $73 \%$. Mit einer Reliabilität von $\kappa=0,68$, kann die Übereinstimmung nach Landis und Koch (1977) als substantiell eingestuft werden. Entsprechend der Empfehlung von Frankfort-Nachmias und Nachmias (1996) wurden im nächsten Schritt die Codes der beiden Raterinnen verglichen und alle Differenzen aufgelöst, indem eine Einigung erzielt wurde.

\section{Ergebnisse}

\subsection{Metakognitive Strategien}

Die deskriptiven Ergebnisse zeigen, dass die Studierenden $(N=118)$ zu beiden Messzeitpunkten im Mittel mit $M=7,9(S D=0,70)$ und $M=7,6(S D=0,86)$ hohe Werte auf der Skala Wissen zu metakognitiven Strategien aufwiesen. Die Häufigkeit der Anwendung metakognitiver Strategien lag zu beiden Messzeitpunkten mit $M=6,6$ $(S D=0,84)$ und $M=6,6(S D=0,95)$ über dem mittleren Bereich der Skala. Die Gruppenmittelwerte und deren Standardabweichungen sind in Abb. 4 dargestellt. Entgegen der Hypothese, dass die App META zu einer Steigerung des Wissens zu metakognitiven SRL-Strategien führt, zeigte sich eine Abnahme des Wissens über die Zeit hinweg. Das Ergebnis der post-hoc Kovarianzanalyse mit Messwiederholung zeigte einen mittleren Zeiteffekt $\left(F(1,77,4)=7,233, p=0,009\right.$ (2-seitig), $\left.\eta_{p}^{2}=0,12\right)$. Einen signifikanten Unterschied zwischen VG und KG gab es jedoch nicht (Zeit $\times$ Gruppe: $F(1,175,2)=0,497, p=0,482$ (2-seitig), $\left.\eta_{p}^{2}=0,01\right)$. Bezüglich der Häufigkeit der Anwendung metakognitiver SRL-Strategien (s. Abb. 4) zeigte sich weder ein signifikanter Effekt über die Zeit hinweg, noch für den Vergleich zwischen VG und KG (Zeit: $F(1,263,5)=0,377, p=0,270$ (1-seitig), $\eta_{p}^{2}<0,01$; Zeit $\times$ Gruppe: $F(1,180,3)=0,590, p=0,443$ (2-seitig), $\left.\eta_{p}^{2}=0,01\right)$. Bezüglich der Kontrollvariable Studienrichtung sowie der Kovariaten Nutzungsintensität und Nutzungszeitraum zeigten sich ebenfalls keine signifikanten Effekte.
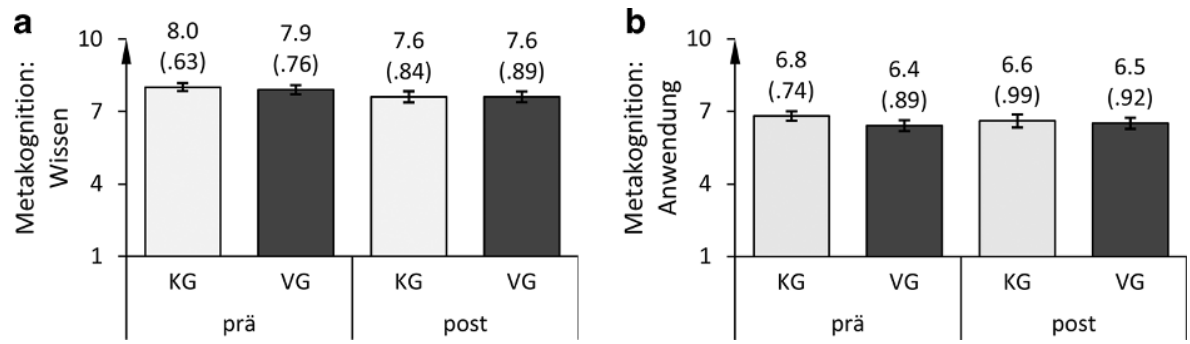

Abb. 4 Metakognition im Längsschnitt (prä-post) mit Fehlerbalken des 95-prozentigen Konfidenzintervalls für den Mittelwert: a Durchschnittliches Wissen zu metakognitiven Strategien „Sehr gering“ (1) bis „Sehr hoch“ (10), b durchschnittliche Häufigkeit der Anwendung von metakognitiven Strategien „Nie“ (1) bis „Immer“ (10). ( $V G$ Versuchsgruppe, $K G$ Kontrollgruppe) 


\subsection{Motivation}

In Hinblick auf die Motivation zum Schreiben der Bachelorarbeit, die im EMoTracker in der App abgefragt wurde, lagen von 32 Studierenden der beiden Studienrichtungen insgesamt 201 Einträge vor. Sechs (18,7\%) Personen machten einen Eintrag, 19 (59,4\%) Personen zwei bis sechs Einträge und sieben $(21,9 \%)$ Personen 12 bis 29 Einträge (s. zusätzliches Onlinematerial C). Mit einem Mittelwert von 2,30 $(S D=1,25)$, gaben die Studierenden eine durchschnittliche Motivation bezüglich des Schreibens der Bachelorarbeit an.

Zur Berechnung der linearen Wachstumsmodelle wurden nur jene Daten der sieben Studierenden mit mindestens 12 Einträgen herangezogen, die im Zeitraum von einem halben bis 92 Tagen gemacht wurden. Ausgehend von einem Nullmodell wurden die festen und zufälligen Effekte schrittweise modelliert. Die Modellkennwerte sind in Tab. 2 zusammengefasst. Das Nullmodell zeigte einen Grand-Mean der durchschnittlichen Motivation von 2,42. Als nächstes wurde ein Random Intercept Only Modell berechnet (Modell 2). Das nichtsignifikante Ergebnis der zufälligen Konstante $(0,097)$ bedeutet, dass sich die Motivation der Studierenden im Mittel nicht voneinander unterschied (Wald $Z=1,139, p=0,255$ ). Im dritten Schritt wurde ein Random Slope Only Modell berechnet (Modell 3), um personenspezifische Steigungen zu modellieren. Die Ergebnisse zeigen, dass über die Zeit hinweg insgesamt eine Zunahme an Motivation erfolgte $(t(33,3)=2,131, p=0,041)$. Diese unterschied sich jedoch nicht signifikant zwischen den Studierenden (Wald $Z=0,148, p=0,883$ ). Ein Random Intercept Random Slope Modell konnte aufgrund nicht erreichter Konvergenz durch Singularität der Hesse-Matrix nicht berechnet werden.

Tab. 2 Veränderung der Motivation beim Schreiben der Bachelorarbeit, Modellparameter der linearen Wachstumsmodelle

\begin{tabular}{llll}
\hline Parameter & $\begin{array}{l}\text { Modell 1 } \\
\text { Nullmodell }\end{array}$ & $\begin{array}{l}\text { Modell 2 } \\
\text { Random Intercept Only }\end{array}$ & $\begin{array}{l}\text { Modell 3 } \\
\text { Random Slope Only }\end{array}$ \\
\hline -2LL(df) & $424,117(2)$ & $419,978(3)$ & $419,672(4)$ \\
AIC & 428,118 & 425,978 & 427,672 \\
$\Delta \chi^{2}(\Delta \mathrm{df})^{\mathrm{a}}$ & - & $4,139^{*}(1)$ & $0,306(1)$ \\
Feste Effekte & & & \\
Konstante $(S E)$ & $2,42^{* * *}(0,105)$ & $2,47^{* * *}(0,156)$ & $2,12^{* * *}(0,171)$ \\
Zeit $(S E)$ & - & - & $0,00852^{*}(0,00400)$ \\
Zufällige Effekte & & & \\
Konstante $(S E)$ & - & $0,097(0,085)$ & - \\
Zeit $(S E)$ & - & - & $0,00000269(0,0000182)$ \\
Residuum $(S E)$ & $1,46^{* * *}(0,179)$ & $1,35^{* * *}(0,170)$ & $1,40^{* * *}(0,175)$ \\
\hline
\end{tabular}

a Änderung gegenüber dem vorherigen Modell ${ }^{*} p \leq 0,05 ;{ }^{* * *} p \leq 0,001$ 


\subsection{Attribution}

Die Mittelwerte in Tab. 3 zeigen, dass die Studierenden zu beiden Messzeitpunkten sowohl ihren Erfolg als auch ihren Misserfolg beim Schreiben einer kleinen wissenschaftlichen Arbeit in hohem Maße auf intern/variable Ursachen zurückführten. Der extern/variablen Attribution bei Erfolg stimmten die Studierenden im Mittel am geringsten zu. Tendenziell war die absolute mittlere Differenz der Mittelwerte im prä-post-Vergleich für eine externe Attribution höher als für eine interne.

Die Ergebnisse der Kovarianzanalysen mit Messwiederholung zeigten bei der Ursachenzuschreibung für Erfolg bei der Bachelorarbeit eine signifikante Abnahme der intern/variablen Attribution $\left(F(1,69,9)=4,268, p=0,043, \eta_{p}^{2}=0,08\right)$. Die extern/variable Erfolgsattribution stieg hingegen signifikant über die Zeit an $\left(F(1,160,9)=6,512, p=0,011, \eta_{p}^{2}=0,08\right)$. Signifikante Veränderungen zwischen VG und $\mathrm{KG}$ zeigten sich für die beiden Erfolgsattributionen jedoch nicht (Zeit $\times$ Gruppe intern/variabel: $F(1,76,1)=1,130, p=0,291, \eta_{p}^{2}=0,03$; extern/variabel: $\left.F(1,162,8)=1,259, p=0,263, \eta_{p}^{2}=0,02\right)$.

Bei der Zustimmungstendenz zu einer extern/stabilen Misserfolgsattribution konnte eine signifikante Zunahme über die Zeit festgestellt werden $(F(1,325,1)=$ $\left.6,164, p=0,014, \eta_{p}^{2}=0,07\right)$, jedoch auch hierbei keine signifikante Veränderung zwischen KG und VG $\left(F(1,105,6)=1,532, p=0,218, \eta_{p}^{2}=0,03\right)$. Für die anderen Erfolgs- und Misserfolgsattributionen waren keine signifikanten Haupt- und Interaktionseffekte feststellbar (Erfolg: Zeit $p$ 's $\geq 0,168$, Zeit $\times$ Gruppe $p$ 's $\geq 0,198$; Misserfolg: Zeit $p$ 's $\geq 0,230$, Zeit $\times$ Gruppe $p$ 's $\geq 0,461)$. Für die Kontrollvariable

Tab. 3 Mittelwert (Standardabweichung) und mittlere Differenz der Zustimmungstendenz zu unterschiedlichen Attributionen bei Erfolg und bei Misserfolg im Längsschnitt

\begin{tabular}{|c|c|c|c|c|c|c|c|}
\hline \multirow[t]{2}{*}{ Gruppe } & \multirow{2}{*}{$\begin{array}{l}\text { Lokation/ } \\
\text { Stabilität }\end{array}$} & \multicolumn{2}{|l|}{ Prä } & \multicolumn{2}{|l|}{ Post } & \multicolumn{2}{|c|}{ Mittl. Differenz } \\
\hline & & Stabil & Variabel & Stabil & Variabel & Stabil & Variabel \\
\hline \multicolumn{8}{|c|}{ Attribution bei Erfolg: } \\
\hline VG & Intern & $\begin{array}{l}5,2 \\
(4,52)\end{array}$ & $\begin{array}{l}9,1 \\
(2,78)\end{array}$ & $\begin{array}{l}6,0 \\
(4,49)\end{array}$ & $\begin{array}{l}7,9 \\
(3,82)\end{array}$ & 0,8 & $-1,2$ \\
\hline KG & & $\begin{array}{l}5,2 \\
(4,53)\end{array}$ & $\begin{array}{l}9,6 \\
(1,82)\end{array}$ & $\begin{array}{l}4,9 \\
(4,49)\end{array}$ & $\begin{array}{l}8,9 \\
(3,00)\end{array}$ & $-0,3$ & $-0,7$ \\
\hline VG & Extern & $\begin{array}{l}5,6 \\
(4,53)\end{array}$ & $\begin{array}{l}3,8 \\
(4,19)\end{array}$ & $\begin{array}{l}6,5 \\
(4,40)\end{array}$ & $\begin{array}{l}5,7 \\
(4,52)\end{array}$ & 0,9 & 1,9 \\
\hline $\mathrm{KG}$ & & $\begin{array}{l}3,9 \\
(4,22)\end{array}$ & $\begin{array}{l}2,4 \\
(3,24)\end{array}$ & $\begin{array}{l}4,9 \\
(4,47)\end{array}$ & $\begin{array}{l}3,4 \\
(4,00)\end{array}$ & 1,0 & 1,0 \\
\hline \multicolumn{8}{|c|}{ Attribution bei Misserfolg: } \\
\hline VG & Intern & $\begin{array}{l}3,3 \\
(3,97)\end{array}$ & $\begin{array}{l}8,6 \\
(3,25)\end{array}$ & $\begin{array}{l}3,4 \\
(3,96)\end{array}$ & $\begin{array}{l}7,9 \\
(3,82)\end{array}$ & 0,1 & $-0,7$ \\
\hline KG & & $\begin{array}{l}3,1 \\
(3,81)\end{array}$ & $\begin{array}{l}8,3 \\
(3,53)\end{array}$ & $\begin{array}{l}3,9 \\
(4,20)\end{array}$ & $\begin{array}{l}8,4 \\
(3,48)\end{array}$ & 0,8 & 0,1 \\
\hline VG & Extern & $\begin{array}{l}3,4 \\
(4,00)\end{array}$ & $\begin{array}{l}2,0 \\
(2,88)\end{array}$ & $\begin{array}{l}5,2 \\
(4,50)\end{array}$ & $\begin{array}{l}2,8 \\
(3,60)\end{array}$ & 1,8 & 0,8 \\
\hline KG & & $\begin{array}{l}3,1 \\
(3,84)\end{array}$ & $\begin{array}{l}2,0 \\
(2,91)\end{array}$ & $\begin{array}{l}4,2 \\
(4,31)\end{array}$ & $\begin{array}{l}2,8 \\
(3,61)\end{array}$ & 1,1 & 0,8 \\
\hline
\end{tabular}

$V G$ Versuchsgruppe, $K G$ Kontrollgruppe 
Studienrichtung und die beiden Kovariaten Nutzungsintensität und Nutzungszeitraum zeigten sich keine signifikanten Effekte.

\subsection{Leistung und Studienerfolg}

Der Notendurchschnitt der letzten drei absolvierten Vorlesungs-Prüfungen lag zum ersten Erhebungszeitpunkt auf der fünfstufigen österreichischen Notenskala von $1=$, ,Sehr gut" bis $5=$,Nicht genügend“" in der VG und KG jeweils bei $M=2,4$ $\left(S D_{\mathrm{VG}}=0,77 ; S D_{\mathrm{KG}}=0,71\right)$. Zum zweiten Erhebungszeitpunkt lag der Notendurchschnitt in der VG ebenfalls bei $M=2,4(S D=0,85)$, in der KG bei $M=2,6(S D=0,94)$. In der Kovarianzanalyse mit Messwiederholung zeigte sich weder ein signifikanter Haupteffekt über die Zeit hinweg $\left(F(1,99,0)=1,063, p=0,305\right.$ (2-seitig), $\eta_{p}^{2}=$ $0,02)$, noch ein signifikanter Interaktionseffekt Zeit $\times$ Gruppe $(F(1,102,8)=1,523$, $p=0,220$ (2-seitig), $\left.\eta_{p}^{2}=0,03\right)$.

Der Notenmittelwert der letzten kleinen wissenschaftlichen Arbeit lag zu Beginn des Semesters (prä) für die VG bei $M=1,7(S D=0,82)$, bei der KG bei $M=1,8(S D=0,75)$. Zum Ende des Semesters (post) war der Notendurchschnitt für KG und VG jeweils um ein Zehntel niedriger $\left(M_{\mathrm{KG}}=1,7, S D_{\mathrm{KG}}=0,84 ; M_{\mathrm{VG}}=1,6\right.$, $\left.S D_{\mathrm{VG}}=0,77\right)$. Signifikante Mittelwertsunterschiede konnten jedoch nicht beobachtet werden (Zeit: $F(1,109,7)=1,122, p=0,292$ (2-seitig), $\eta_{p}^{2}=0,02$; Zeit $\times$ Gruppe: $F(1,736,6)=0,293, p=0,588$ (2-seitig), $\left.\eta_{p}^{2}<0,01\right)$. Für die Kontrollvariable Studienrichtung und die beiden Kovariaten Nutzungsintensität und Nutzungszeitraum zeigten sich keine signifikanten Effekte.

Bezüglich der Bachelorarbeit selbst zeigte sich im Follow-up $(N=46)$, dass der Großteil der Studierenden (62\%) mit einem Notendurchschnitt von $M=1,45$ $(S D=0,63)$ eine sehr gute Note auf die Bachelorarbeit erhielt. 31,6\% der Studierenden bekamen eine 2 (,gut") und 6,4\% eine 3 (,,befriedigend"). Die Studierenden der Psychologie $(M=1,45, S D=0,63)$ und der Wirtschaftswissenschaften $(M=1,47$, $S D=0,64)$ unterschieden sich im Mittel nicht bezüglich ihrer Leistung in der Bachelorarbeit $(t(42)=-0,091, p=0,928)$. Über die Studiengänge hinweg erzielte die VG einen Notendurchschnitt von $M=1,35(S D=0,56)$ und die KG einen Notendurchschnitt von $M=1,61(S D=0,70)$; dieser Unterschied war jedoch nicht signifikant $(t(44)=1,108, p=0,274)$. Im Mittel war es den Studierenden sehr wichtig eine gute Leistung in der Bachelorarbeit zu erbringen $(M=8,8, S D=1,86)$.

\subsection{Nutzung der App - Analyse der Log-Files}

Bei der Analyse der Log-Files zum Nutzungsverhalten zeigte sich eine rapide Abnahme in der Nutzung der App nach Beginn der Intervention. $61 \%$ (39) der Studierenden loggten sich einmal in die App ein, $48 \%$ erreichten mindestens das erste von insgesamt fünf Leveln. Am Ende des Semesters nutzten $38 \%$ (25) der Studierenden die App noch häufig, wobei $20 \%$ (13) das höchste Level erreichten - also alle Module absolvierten und Zeitplan sowie Tracker regelmäßig nutzten.

Für jene Studierenden, die sich mindestens einmal eingeloggten, lag der Median des Nutzungszeitraums über das Semester hinweg (bis zum letzten Login) bei 67,1 Tagen. 39 Personen nutzen die App im Mittel (Median) an zehn verschiedenen 
Tagen. Dabei klickten die Studierenden durchschnittlich Mdn=91-mal auf SRLrelevante Komponenten der App.

Das Tagebuch (My Diary) wurde von 34 Studierenden ein- bis 39-mal aufgerufen. 24 Studierende machten im Mittel Mdn=4 Einträge, das Maximum lag bei 35 Einträgen. Im Mittel (Median) betrug die Bearbeitungsdauer des Tagebuchs bei den 34 Studierenden etwa zehn Minuten. Eine Übersicht zum Nutzungsverhalten der Studierenden $(N=39)$, kann dem zusätzlichen Onlinematerial C entnommen werden.

Ein genauer Blick auf die Daten zeigt allerdings, dass acht Studierende das Tagebuch mindestens $12 \mathrm{~h}$ am Stück geöffnet hatten (Max $=23 \mathrm{~h})$. Da nicht kontrolliert werden konnte, wie lange die Studierenden das Tagebuch tatsächlich bearbeiteten, ist von einer Verzerrung der Daten auszugehen. In den Analysen wurden diese Daten deshalb nicht als Kontrollvariable berücksichtigt.

\subsection{Bewertung der Nützlichkeit der App}

Die Ergebnisse der Follow-up-Erhebung zeigen, dass die Studierenden der VG die App im Mittel als eher wenig nützlich wahrgenommen haben $(N=26 ; M=3,8$, $S D=2,52)$ und die App laut eigenen Angaben eher selten nutzten $(M=3,8$, $S D=2,33)$. Bezüglich der einzelnen Funktionen der App bewerteten die Studierenden der VG den Zeitplan $(M=7,5, S D=2,79)$ und die Milestones $(M=6,7$, $S D=3,13)$ als nützlich, wohingegen die Tagebuchfunktion als eher wenig nützlich bewertet wurde $(M=3,0, S D=2,47)$.

Einen differenzierten Einblick in die Gründe für die geringe wahrgenommene Nützlichkeit und die geringe Nutzung der App bieten die Ergebnisse der qualitativen Analyse der offenen Antworten aus der Follow-up-Erhebung (s. zusätzliches Onlinematerial D). Einer der wichtigsten Gründe für die geringe Nutzung und die als gering empfundene Nützlichkeit der App seitens der Studierenden bestand darin, dass die Studierenden bereits eigene Strategien etabliert hatten, mit denen sie die Anforderungen des Studiums erfolgreich meisterten. $18 \%$ der 122 codierten Textsegmente wurden dieser Kategorie zugeordnet. 14\% der Textsegmente bezogen sich auf fehlende Integrations- bzw. Synchronisationsmöglichkeiten der App in den eigentlichen Arbeitsprozess und mit bereits bestehenden Arbeitsressourcen, z. B. durch eine Browseroberfläche (PC). $11 \%$ der Gründe bezogen sich auf die als unpassend empfundene Interventionssituation. Zum einen wurde die Bachelorarbeit von einigen Studierenden als wenig herausfordernd wahrgenommen, zum anderen wurde mehrfach vorgeschlagen, eine solche Intervention besser zu Beginn des Studiums zu implementieren. Ebenfalls $11 \%$ der Gründe für die seltene App-Nutzung fielen in die Kategorie „Komplexität/Aufwand“, die sich darauf bezieht, dass die Struktur der App zu komplex war bzw. dass der mit der Nutzung der App einhergehende Aufwand zu hoch war. Die Kategorie „Konkurrenzfähigkeit“, der $10 \%$ der Textsegmente zugeordnet wurden, bezieht sich auf Mängel in der Umsetzung und Programmierung der App. Die Kategorie umfasst solche Antworten, die die AppGestaltung von META als inferior gegenüber unternehmerisch entwickelter Apps beschreiben. Dazu gehören Anmerkungen zu Attraktivität und Schnelligkeit, aber auch ein fehlendes Alleinstellungsmerkmal gegenüber anderen Apps. Schließlich bezogen sich $9 \%$ der Textsegmente direkt auf das Medium Smartphone-App. Stu- 
dierende gaben hier unter anderem eine generelle Abneigung gegenüber Apps an bzw. bemängelten den Aufwand, den beispielsweise das Eintippen von Texten am Smartphone mit sich bringt.

\section{Diskussion}

Ziel der Studie war es, eine SRL-Intervention zu entwickeln, pilotieren und explorativ zu evaluieren. Durch eine ökonomische und niedrigschwellige Implementierung via App-Format sollten einige Defizite bisheriger SRL-Interventionen im tertiären Sektor überwunden werden. Die Intervention wurde im ökologisch validen Kontext universitärer Bachelorarbeit-Seminare durchgeführt und im Rahmen eines VersuchsKontrollgruppendesigns evaluiert. Insgesamt konnte dabei kein Trainingseffekt nachgewiesen werden. Die App-Intervention konnte keine Verbesserung von Wissen zu und Anwendung von metakognitiven Strategien bewirken (Forschungsfrage 1). Während bei jenen Teilnehmenden, die die App regelmäßig nutzten eine Steigerung der prozessorientierten Arbeitsmotivation verzeichnet werden konnte, zeigte sich über alle Gruppen hinweg eine signifikante Veränderung in Richtung eines sehr ungünstigen Attributionsstils (Forschungsfrage 2). So stieg über die Zeit hinweg die extern/ variable Erfolgsattribution signifikant an, während die intern/variable Erfolgsattribution signifikant abnahm. Für Misserfolg zeigte sich eine signifikante Zunahme extern/stabiler Attribution. Was die Leistung betrifft (Forschungsfrage 3), konnte kein signifikanter Effekt festgestellt werden. Auffallend ist der überdurchschnittlich gute Notendurchschnitt in allen Gruppen. Bezüglich der Forschungsfrage 4 - inwieweit die Studierenden die App-Intervention als nützlich empfanden und nutzten - zeigt sich sowohl objektiv bei der Betrachtung der Nutzungshäufigkeit als auch subjektiv in der Nützlichkeitseinschätzung ein eher negatives Bild.

Zwar scheinen die Ergebnisse auf den ersten Blick erwartungswidrig, tatsächlich finden sich jedoch in einer Reihe von SRL-Interventionen ähnliche Befunde insbesondere bei der Förderung von Self-monitoring mittels Lerntagebüchern (Dignath-van Ewijk et al. 2015; Dörrenbächer und Perels 2016a; Fabriz et al. 2014). So berichten beispielsweise Fabriz et al. (2014) in ihrer SRL-Interventionsstudie von einer Verringerung der Motivation bei Studierenden, Dörrenbächer und Perels (2016a) von einer Verschlechterung hinsichtlich der SRL-Kompetenzen.

Im Folgenden soll unter anderem auf Basis der qualitativ gewonnen Informationen über die Rezeption seitens der Studierenden beleuchtet werden, welche Faktoren für die Ergebnisse verantwortlich sein könnten. Dabei steht die Frage im Zentrum, warum die Studierenden die App zu wenig genutzt haben. Es werden einerseits Toolspezifische Erklärungsfaktoren diskutiert, um Empfehlungen für Interventionsimplementierungen via App abzuleiten. Zum anderen werden konzeptionelle Aspekte der Intervention diskutiert, die unabhängig vom gewählten Interventionstool zu beachten sind. 


\subsection{Tool-spezifische Erklärungsfaktoren}

Empirisch konnte vielfach belegt werden, dass die Auseinandersetzung mit neuen Lerntechniken zusätzlich zur eigentlichen Aufgabe eine kognitive Belastung für das Arbeitsgedächtnis bedeutet (Kalyuga 2007, 2003; Lan 2005). SRL ist ein hochkomplexer und vielschichtiger Prozess, dessen Sub-Komponenten nicht isoliert, sondern in Interaktion miteinander zu verstehen sind (für einen Überblick s. Panadero 2017). In der Entwicklung der App wurde nicht nur versucht verschiedene zentrale SRLStrategien inhaltlich zur berücksichtigen, sondern die Verschränkung zwischen metakognitiven und motivationalen Faktoren auch in der App-Gestaltung umzusetzen (z.B. durch die hierarchisch strukturierte Darbietung von SRL-Strategien und den entsprechenden Sub-Strategien). Kalyuga et al. (2003) postulieren jedoch, dass diese element interactivity das Arbeitsgedächtnis in hohem Maße beansprucht. In der vorliegenden Intervention ist nicht nur der Interventionsinhalt (SRL), sondern auch das Interventionsmedium (Interface der App) von hoher element interactivity gekennzeichnet. Der Versuch, die Verschränkungen metakognitiver und motivationaler Strategien im Aufbau der App umzusetzen und so erfahrbar zu machen könnte demnach den gegenteiligen Effekt erwirkt haben und anstelle intuitiven Erlebens eher zu Überwältigung geführt haben. Im Hinblick auf die genannten Gründe für die geringe Nutzung der App seitens der Studierenden wird dies dadurch gestützt, dass die hohe Komplexität der App und der damit verbundene Aufwand der Nutzung bemängelt wurde. Während die Komplexität, die mit dem Konstrukt SRL einhergeht, auf computerbasierte Interventionen bereits erfolgreich transferiert werden konnte (Azevedo und Cromley 2004; Bellhäuser et al. 2016; Hadwin und Winne 2001), scheint ein essentielles Merkmal von Apps darin zu liegen, dass sie sich auf wenige, klar umrissene Funktionen beschränken müssen um die Usability zu gewährleisten. Wie groß die Unterschiede zwischen einer Interventionsimplementierung via PC und via Smartphone sind wird dadurch deutlich, dass der PC von den teilnehmenden Studierenden als Arbeitsmedium gesehen wurde, während das Smartphone in erster Linie mit Freizeit assoziiert wurde. Da laut des split attention effects (Kalyuga et al. 2003) die Integration zweier Informationsquellen mit hoher kognitiven Anstrengung einhergeht, ist davon auszugehen, dass Informationsquellen die nicht einmal auf demselben Medium lokalisiert sind (PC als Arbeitstool vs. Smartphone als Interventionstool), eine große Belastung der kognitiven Ressourcen mit sich bringt. Einige Studien (für eine Übersicht s. Kalyuga 2007) konnten bereits Evidenzen dafür liefern, dass der Einfluss der Komplexität und Struktur von Hypermedia auf die Lernergebnisse vom Kompetenzniveau der Lernenden mediiert wird. Um eine Aussage darüber treffen zu können, welche Lernenden von welchen Gestaltungsmerkmalen in Hypermedia am besten profitieren, bedarf es jedoch weiterer Forschung. Vor dem Hintergrund, dass die Bachelorarbeit generell als wenig herausfordernd empfunden wurde, steht der Aufwand der Integration der verschiedenen Tools und Ressourcen kaum im Verhältnis zum Nutzen für das Schreiben der Bachelorarbeit. Um dem split attention effect entgegen zu wirken, muss eine App also perfekt in die bereits bestehenden Arbeitsressourcen und -tools integriert sein. Das bedeutet einerseits, dass ein Zugang zu den Informationen auch über das Arbeitsmedium PC gewährleistet sein muss, andererseits müssen die Informationen, die auf 
verschiedenen Medien lokalisiert sind, automatisch synchronisiert und damit zentral gebündelt werden.

\subsection{Inhaltlich konzeptionelle Erklärungsfaktoren}

Eine Strategie um den Aufwand durch die Anwendung bestimmter Lernstrategien zu rechtfertigen, ist die Verdeutlichung des Nutzens der Intervention für die Studierenden (Dörrenbächer und Perels 2016a; Lan 2005). Da in der vorliegenden Studie sowohl bei der Vorstellung der einzelnen Funktionen der App als auch der SRLSubstrategien stets über Nutzen und Effektivität informiert wurde und der Bachelorarbeit ein hoher subjektiver Wert beigemessen wurde, könnte man im Sinne der Erwartungs-Wert-Theorie (Wigfield und Eccles 2000) davon ausgehen, dass die Studierenden über eine hohe Leistungsmotivation verfügten. Zwar wird in der Literatur empfohlen den Nutzen für die Studierenden hervorzuheben, die reine Information über den Nutzen bedeutet allerdings noch nicht, dass dieser individuell tatsächlich erkannt wird. So zeigen einige Studien, dass Studierende ihr eigenes Wissen - vor allem hinsichtlich Metakognition - stark überschätzen (Cohen 2012; Dunlosky und Rawson, 2012) und paradoxerweise gerade bei schwierigen Aufgaben dazu tendieren, die Effektivität von Self-Monitoring zu bezweifeln (Lan 2005). Vor diesem Hintergrund könnten die Ergebnisse dahingehend interpretiert werden, dass eine überzogene Selbsteinschätzung zu Beginn des Semesters angesichts der Herausforderungen der Bachelorarbeit, die ein hohes Maß an Selbstregulation erfordert (vgl. Dresel et al. 2015), nach unten korrigiert wurde. Die signifikante Abnahme des selbstberichteten Wissens zu metakognitiven SRL-Strategien in Verbindung mit der signifikanten Zunahme extern/stabiler Misserfolgs- und extern/variabler Erfolgsattribution sowie der signifikanten Abnahme intern/variabler Erfolgsattribution, deutet auf einen Verlust des Kontrollgefühls über das Arbeitsergebnis hin, was Konsequenz der anfänglichen Selbstüberschätzung sein könnte. Die objektiv hohen Leistungen - also die Noten auf die Bachelorarbeit - scheinen der Annahme zu widersprechen, dass die Studierenden sich zu Beginn des Semesters überschätzten. Bei Betrachtung des Notendurchschnitts über alle Bachelorarbeit-Seminare, zeigt sich jedoch kaum Varianz in der Notengebung. Tatsächlich muss hinterfragt werden, inwieweit Noten mit so geringer Varianz als abhängige Variable für Veränderungen der SRLKompetenzen fungieren können. So könnte die heterogene Befundlage bezüglich des Zusammenhangs zwischen SRL und Leistung nicht nur in messmethodischen Defiziten von SRL, sondern auch in der Messung von Leistung begründet sein.

Foerst et al. (2017) konnten zeigen, dass Studierende über ein breites Wissen bezüglich SRL verfügen, dieses jedoch nicht anwenden. Es scheint durchaus möglich, dass das umfangreiche deklarative Wissen bezüglich SRL dazu führt, dass nicht zwischen deklarativem, prozeduralem und konditionalem Wissen differenziert wird. Es sind jedoch das know-how und know-when, die den Transfer des deklarativen Wissens hin zur Anwendung ermöglichen (Dresel et al. 2015). Dunlosky und Rawson (2012) konnten zeigen, dass eine Selbstüberschätzung von Studierenden zu schlechteren Leistungen führen kann, weshalb die Fähigkeit zur akkuraten Beurteilung des eigenen Lernvorgangs als ein Interventionsziel fokussiert werden sollte. Um Studierenden den Nutzen einer SRL-Intervention nahe zu bringen müsste also in einem 
ersten Schritt insbesondere an einer realistischen Selbsteinschätzung gearbeitet werden. Die Zunahme an Motivation, wie sie bei jenen Teilnehmenden zu finden ist die die App tatsächlich regelmäßig genutzt haben, könnte ein Hinweis dafür sein, dass die Intervention einen positiven Einfluss erzielen kann, sofern die regelmäßige Nutzung gewährleistet ist und diese von den Studierenden angenommen wird.

Die Implementierung von Self-Monitoring führt häufig zu Widerstand seitens der Studierenden (Dignath-van Ewijk et al. 2015). Einerseits kann dies mangelnder Motivation zugeschrieben werden (Fabriz et al. 2014), andererseits stellt Self-Monitoring eine zusätzliche kognitive Belastung dar (Dignath van Ewjik et al. 2015), die Studierende teils aus dem Konzept bringt und von der eigentlichen Aufgabe ablenkt, anstatt sie zu unterstützen (Winne 1995). Aus den qualitativen Daten geht hervor, dass die Studierenden bereits eigene Strategien etabliert haben, mit denen sie bisher erfolgreich das Studium bewältigen konnten. Laut Kalyuga et al. (2003) kann bei Studierenden, die bereits über ein hohes Wissen im Interventionsthema verfügen ein Konflikt zwischen den eigenen Schemata (hier also den eigenen Lernstrategien) und der externen Anleitung (hier die durch die App angeleiteten Lernstrategien) entstehen. In Folge wird die Quelle eliminiert, die redundante Information enthält (redundancy effect). Besteht also eine Diskrepanz zwischen den eigenen Lernstrategien und den durch die App angeleiteten Lernstrategien, wäre die absichtliche Abwendung von der App nicht nur die selbstwertschützendere, sondern auch die ökonomischere Option zur Dissonanzreduktion.

Einen weiteren Erklärungs- und Interventionsansatz, bietet der Aptitude-treatment-interaction approach (Snow et al. 1996), der die Anpassung der Interventionsmethode an die individuellen Fähigkeiten der Rezipientinnen und Rezipienten ins Zentrum rückt. So kommen Studien, die die unterschiedlichen Voraussetzungen hinsichtlich SRL-Kompetenz und Motivation explizit in ihren Analysen berücksichtigt haben zu dem Schluss, dass unterschiedliche Kompetenzniveaus der Teilnehmenden zu Beginn der Studie einen signifikanten Einfluss auf die Effektivität der Intervention ausüben (Dörrenbächer und Perels 2016b; Fabriz et al. 2014). Diese Erkenntnisse wurden bisher allerdings kaum in Interventionen umgesetzt. Eine individuelladaptive Gestaltung eines klassischen SRL-Trainings wäre aufwand- und ressourcentechnisch kaum umzusetzen. Die Stärke einer Implementierung via App liegt in der Möglichkeit, aus einem umfassenden Pool an Strategien und Übungen eine breit implementierbare, individuell-adaptive Intervention zur Verfügung zu stellen. Das Ziel der vorliegenden Pilotstudie lag in der Gewinnung von ersten Informationen zur Effektivität einer SRL-App und zum Nutzungsverhalten seitens der Studierenden, weshalb die Implementierung adaptiver Logarithmen, die die Inhalte auf das individuelle Profil der/des Studierenden abstimmt, den Rahmen der Studie gesprengt hätte. Auf Basis der Erkenntnisse der vorliegenden Studie sollte darin jedoch der nächste Forschungsfokus liegen. 


\subsection{Implikationen und Limitationen der Studie}

\subsubsection{Implikationen für Forschung und Praxis}

Zusammenfassend lassen sich aus den Ergebnissen der vorliegenden Studie einige Empfehlungen für die Entwicklung von SRL-Interventionen sowie für die Umsetzung via App-Format ableiten. Zu Beginn einer Intervention muss gewährleistet sein, dass die Teilnehmenden den Mehrwert dieser erkennen. In Bezug auf SRL kann dies durch eine überhöhte Selbsteinschätzung der eigenen Kompetenzen kompromittiert sein. Es empfiehlt sich daher, eine profilorientierte Rückmeldung über Stärken und Schwächen der einzelnen Teilnehmenden zu Beginn der Intervention zu realisieren. Grundsätzlich stellt sich die Frage, wie nutzbringend klassische „one-for-all“ Interventionen sind. So besteht die Relevanz von Apps als Interventionstool neben der flächendeckenden Implementierbarkeit vor allem darin, anstelle eines Gießkannenprinzips den unterschiedlichen Kompetenzniveaus der Nutzerinnen und Nutzer gerecht werden zu können. In Zukunft könnte eine App entwickelt werden, die auf Basis eines integrierten Prätests das Kompetenzniveau der Studierenden ermittelt und rückmeldet. Auf Basis dieses Profils könnte eine adaptive App die Studierenden genau dort fördern, wo sie Defizite aufweisen. So könnten Konflikte zwischen den Schemata der Studierenden und dem Interventionsinhalt sowie redundante Informationen vermieden werden. Zwar ist die Entwicklung einer solchen App mit hohem Aufwand und Kosten verbunden, doch wäre es möglich, eine im Lehrkontext breit einsetzbare Intervention zu entwickeln, die dennoch maximal spezifisch auf die individuellen Voraussetzungen der Studierenden angepasst ist.

Durch die Ergebnisse der Studie wird deutlich, dass die hohe Komplexität des Themas SRL der Usability einer App, die als alleiniges Interventionstool genutzt wird, entgegensteht. Zukünftige Forschung sollte sich der Frage widmen, inwieweit eine App - die auf einige klar umrissene Funktionen beschränkt ist - zur Effektivität einer SRL-Intervention beitragen kann. Dabei müssen Synchronisationsmöglichkeiten mit den bestehenden Ressourcen gewährleistet sein und dadurch eine Integration in den Arbeitsprozess realisiert werden. Im Gegensatz zu einer relativ unabhängig vom eigentlichen Seminar laufenden App-Intervention, wäre somit auch eine stärkere Verschränkung zwischen Intervention und Studienalltag gegeben, was sowohl Integration als auch Transfer des Gelernten unterstützen könnte.

\subsubsection{Limitationen}

Mit Blick auf die ökologische Validität wurde die Intervention in regulären Bachelorarbeit-Seminaren durchgeführt, wodurch sich einige Einschränkungen ergaben. Bedingt durch formale Vorgaben konnte keine Verpflichtung zur Teilnahme realisiert werden. Trotz ausreichender Stichprobengröße im Studiendesign, führte die Freiwilligkeit der Teilnahme neben möglichen Selektionseffekten zu einem hohen Dropout während der Interventionsstudie. Dies resultierte wiederum in Einschränkungen der Analysemöglichkeiten aufgrund geringer Sub-sample-Größen. Um eine Verzerrung bei der inferenzstatistischen Schätzung durch die insgesamt $39 \%$ fehlenden Fälle im Längsschnitt zu reduzieren wurde unter der Annahme, dass den fehlenden 
Werten ein Missing-At-Random-Prozess zugrunde liegt, eine multiple Imputation durchgeführt.

Da es sich bei den Items zur Attribution von Erfolg und Misserfolg um Einzelitems entsprechend des Vierfelderschemas der Attribution nach Weiner (1985) handelt (Fähigkeit, Anstrengung, Aufgabenschwierigkeit, Pech/Glück), die theoretisch von einander unabhängig sind, sind Aussagen bezüglich der Reliabilität nicht möglich bzw. sinnvoll.

Bezüglich der Messung von SRL - insbesondere metakognitiver Strategien - gibt es diverse Ansätze, die unterschiedliche Vor- und Nachteile mit sich bringen (Spörer und Brunstein 2006; Veenman et al. 2006). Grundsätzlich besteht bei Selbstberichtsverfahren die Gefahr einer Verzerrung des Antwortverhaltens in Richtung sozialer Erwünschtheit. Spezifische Kritik an SRL-Fragebögen richtet sich zudem vor allem auf die Nicht-Berücksichtigung der Anwendungskomponente und Situationsspezifität von SRL. In der vorliegenden Studie wurde daher bewusst ein Fragebogen (SRLQuAK, Foerst et al. 2017) eingesetzt, der nicht nur das Wissen zu, sondern auch die Anwendung von metakognitiven SRL-Strategien in einer konkreten Studiensituation erfragt. Auch wenn die Nachteile eines Selbstbericht-Verfahrens nicht völlig überwunden werden können, lassen die diskrepanten Ergebnisse zwischen Wissen zu und Anwendung von metakognitiven SRL-Strategien keine Verzerrung im Sinne sozialer Erwünschtheit vermuten. Dies wird auch durch die Angaben zur seltenen Nutzung der App sowie der entsprechenden Gründe gestützt.

Trotz oder gerade aufgrund der ausbleibenden Effekte und der genannten Einschränkungen, kann die vorliegende Pilotstudie wichtige Impulse für künftige Forschung bieten. Eine Anpassung der Interventionstools an die gesellschaftliche Entwicklung und fortschreitende Technologisierung ist vermutlich unumgänglich. Wie diese Studie aber vermuten lässt, geht es um mehr als den technisch ansprechenden Transfer bisheriger Interventionsansätze auf Apps und Smartphones.

Funding Open access funding provided by University of Vienna.

Open Access Dieser Artikel wird unter der Creative Commons Namensnennung 4.0 International Lizenz (http://creativecommons.org/licenses/by/4.0/deed.de) veröffentlicht, welche die Nutzung, Vervielfältigung, Bearbeitung, Verbreitung und Wiedergabe in jeglichem Medium und Format erlaubt, sofern Sie den/die ursprünglichen Autor(en) und die Quelle ordnungsgemäß nennen, einen Link zur Creative Commons Lizenz beifügen und angeben, ob Änderungen vorgenommen wurden.

\section{Literatur}

Artelt, C., \& Wirth, J. (2014). Kognition und Metakognition. In T. Seidel \& A. Krapp (Hrsg.), Lehrbuch Pädagogische Psychologie (S. 167-192). Weinheim: Beltz.

Azevedo, R. (2005). Computer environments as Metacognitive tools for enhancing learning. Educational Psychologist, 40(4), 193-197. https://doi.org/10.1207/s15326985ep4004_1.

Azevedo, R., \& Cromley, J.G. (2004). Does intervention on self-regulated learning facilitate students' learning with hypermedia? Journal of Educational Psychology, 96(3), 523-535. https://doi.org/10. 1037/0022-0663.96.3.523.

Bail, F. T., Zhang, S., \& Tachiyama, G. T. (2008). Effects of a self-regulated learning course on the academic performance and graduation rate of college students in an academic support program. Journal of College Reading and Learning, 39(1), 54-73. https://doi.org/10.1080/10790195.2008.10850312. 
Bellhäuser, H., Lösch, T., Winter, C., \& Schmitz, B. (2016). Applying a web-based training to foster selfregulated learning-effects of an intervention for large numbers of participants. The Internet and Higher Education, 31, 87-100. https://doi.org/10.1016/j.iheduc.2016.07.002.

Boekaerts, M. (1997). Self-regulated learning: a new concept embraced by researchers, policy makers, educators, teachers, and students. Learning and Instruction, 7(2), 161-186. https://doi.org/10.1016/ S0959-4752(96)00015-1.

Boekaerts, M. (1999). Self-regulated learning: where we are today. International Journal of Educational Research, 31(6), 445-457. https://doi.org/10.1016/S0883-0355(99)00014-2.

Britton, B. K., \& Glynn, S. M. (1989). Mental management and creativity. In J. A. Glover, R. R. Ronning \& C. R. Reynolds (Hrsg.), Handbook of creativity. Perspectives on individual differences (S. 429-440). Boston: Springer.

Briz-Ponce, L., \& García-Peñalvo, F. J. (2015). An empirical assessment of a technology acceptance model for Apps in medical education. Journal of Medical Systems, 39(11), 176. https://doi.org/10.1007/ s10916-015-0352-X.

Briz-Ponce, L., Juanes-Méndez, J. A., García-Peñalvo, F. J., \& Pereira, A. (2016). Effects of mobile learning in medical education: a counterfactual evaluation. Journal of Medical Systems, 40(6), 136. https:// doi.org/10.1007/s10916-016-0487-4.

Butler, D.L., \& Winne, P.H. (1995). Feedback and self-regulated learning: a theoretical synthesis. Review of educational research, 65(3), 245-281. https://doi.org/10.3102/00346543065003245.

Van Buuren, S., \& Groothuis-Oudshoorn, K. (2011). Mice: multivariate imputation by chained equations in R. Journal of Statistical Software, 45. https://doi.org/10.18637/jss.v045.i03.

Carver, C. S., \& Scheier, M. F. (2000). On the structure of behavioral self-regulation. In M. Boekaerts, P. R. Pintrich \& M. Zeidner (Hrsg.), Handbook of self-regulation (S. 41-84). San Diego: Academic Press.

Cohen, M. (2012). The importance of self-regulation for college student learning. College Student Journal, 46(4), 892-902.

Conzemius, A., \& O'Neill, J. (2009). The power of SMART goals: using goals to improve student learning. Bloomington: Solution Tree Press.

Dabbagh, N., \& Kitsantas, A. (2012). Personal learning environments, social media, and self-regulated learning: a natural formula for connecting formal and informal learning. The Internet and Higher Education, 15(1), 3-8. https://doi.org/10.1016/j.iheduc.2011.06.002.

Dignath, C., \& Büttner, G. (2008). Components of fostering self-regulated learning among students. A meta-analysis on intervention studies at primary and secondary school level. Metacognition and Learning, 3(3), 231-264. https://doi.org/10.1007/s11409-008-9029-x.

Dignath, C., Büttner, G., \& Langfeldt, H. (2008). How can primary school students learn self-regulated learning strategies most effectively? A meta-analysis on self-regulation training programmes. Educational Research Review, 3(2), 101-129. https://doi.org/10.1016/j.edurev.2008.02.003.

Dignath-van Ewijk, C., Fabriz, S., \& Büttner, G. (2015). Fostering self-regulated learning among students by means of an electronic learning diary: a training experiment. Journal of Cognitive Education and Psychology, 14(1), 77. https://doi.org/10.1891/1945-8959.14.1.77.

Dörrenbächer, L., \& Perels, F. (2016a). Self-regulated learning profiles in college students: their relationship to achievement, personality, and the effectiveness of an intervention to foster self-regulated learning. Learning and Individual Differences, 51, 229-241. https://doi.org/10.1016/j.lindif.2016.09. 015 .

Dörrenbächer, L., \& Perels, F. (2016b). More is more? Evaluation of interventions to foster self-regulated learning in college. International Journal of Educational Research, 78, 50-65. https://doi.org/10. 1016/j.ijer.2016.05.010.

Dresel, M. (2010). Förderung der Lernmotivation mit attributionalem Feedback. In R. Reimann (Hrsg.), Bildungspsychologie (S. 131-135). Göttingen: Hogrefe.

Dresel, M., Schmitz, B., Schober, B., Spiel, C., Ziegler, A., Engelschalk, T., Jöstl, G., Klug, J., Roth, A., Wimmer, B., \& Steuer, G. (2015). Competencies for successful self-regulated learning in higher education: structural model and indications drawn from expert interviews. Studies in Higher Education, 40(3), 454-470. https://doi.org/10.1080/03075079.2015.1004236.

Dresel, M., Schober, B., \& Ziegler, A. (2005). Nothing more than dimensions? Evidence for a surplus meaning of specific attributions. Journal of Educational Research, 99, 31-44. https://doi.org/10.3200/ JOER.99.1.31-45.

Dunlosky, J., \& Rawson, K. A. (2012). Overconfidence produces underachievement: Inaccurate self evaluations undermine students' learning and retention. Learning and Instruction, 22(4), 271-280. https:// doi.org/10.1016/j.learninstruc.2011.08.003. 
European Commission (2008). The European Qualifications Framework for Lifelong Learning (EQF). Luxembourg: Office for official publications of the European Communities.

Fabriz, S., Dignath-van Ewijk, C., Poarch, G., \& Büttner, G. (2014). Fostering self-monitoring of university students by means of a standardized learning journal-a longitudinal study with process analyses. European Journal of Psychology of Education, 29(2), 239-255. https://doi.org/10.1007/s10212-0130196-z.

Feierabend, S., Plankenhorn, T., \& Rathgeb, T. (2017, November). JIM 2017 Jugend, Information, (Multi-) Media - Basisstudie zum Medienumgang 12- bis 19-Jähriger in Deutschland. Stuttgart: Medienpädagogischer Forschungsverbund Südwest.

Foerst, N.M., Klug, J., Jöstl, G., Spiel, C., \& Schober, B. (2017). Knowledge vs. action: discrepancies in university students' knowledge about and self-reported use of self-regulated learning strategies. Frontiers in psychology, 8, 1288. https://doi.org/10.3389/fpsyg.2017.01288.

Frankfort-Nachmias, C., \& Nachmias, D. (1996). Research methods in the social sciences. London: Arnold.

García-Ros, R., Pérez-González, F., \& Hinojosa, E. (2004). Assessing time management skills as an important aspect of student learning: the construction and evaluation of a time management scale with Spanish high school students. School Psychology International, 25(2), 167-183. https://doi.org/10. $1177 / 0143034304043684$.

Gomes, C. M. A., Golino, H. F., \& Menezes, I. G. (2014). Predicting school achievement rather than intelligence: does metacognition matter? Psychology, 05(09), 1095-1110. https://doi.org/10.4236/psych. 2014.59122.

Hadwin, A.F., \& Winne, P.H. (2001). ConoteS2: a software tool for promoting self-regulation. Educational Research and Evaluation, 7(2-3), 313-334. https://doi.org/10.1076/edre.7.2.313.3868.

Hattie, J., Biggs, J., \& Purdie, N. (1996). Effects of learning skills interventions on student learning: a meta-analysis. Review of Educational Research, 66(2), 99-136. https://doi.org/10.3102/ 00346543066002099.

Kalyuga, S. (2007). Expertise reversal effect and its implications for learner-tailored instruction. Educational Psychology Review, 19(4), 509-539. https://doi.org/10.1007/s10648-007-9054-3.

Kalyuga, S., Ayres, P., Chandler, P., \& Sweller, J. (2003). The expertise reversal effect. Educational Psychologist, 38(1), 23-31. https://doi.org/10.1207/S15326985EP3801_4.

Klein, S., Koch, C. J., \& Kleinmann, M. (2003). Zeitmangament: ein Überblick

Krapp, A., Geyer, C., \& Lewalter, D. (2014). Motivation und Emotion. In T. Seidel \& A. Krapp (Hrsg.), Pädagogische Psychologie (S. 193-222). Weinheim: Beltz.

Lan, W. Y. (2005). Self-monitoring and its relationship with educational level and task importance. Educational Psychology, 25(1), 109-127. https://doi.org/10.1080/0144341042000294921.

Landis, J.R., \& Koch, G. G. (1977). The measurement of observer agreement for categorical data. biometrics, 159-174. https://doi.org/10.2307/2529310.

Landmann, M., \& Schmitz, B. (Hrsg.). (2007). Selbstregulation erfolgreich fördern. Praxisnahe Interventionsprogramme für effektives Lernen. Stuttgart: Kohlhammer.

Macan, T.H. (1994). Time management: test of a process model. Journal of Applied Psychology, 79(3), 381-391. https://doi.org/10.1037/0021-9010.79.3.381.

Mandl, H., \& Friedrich, H. F. (2006). Handbuch Lernstrategien. Göttingen: Hogrefe.

Martin, R., McGill, T., \& Sudweeks, F. (2013). Learning anywhere, anytime: Student motivators for m-learning. Journal of Information Technology Education: Research, 12(1), 51-67. https://doi.org/ $10.28945 / 1771$.

Metzger, C. (2002). Lern- und Arbeitsstrategien. Ein Fachbuch für Studierende an Universitäten und Fachhochschulen. Aarau: Sauerländer.

Panadero, E. (2017). A review of self-regulated learning: six models and four directions for research. Frontiers in psychology, 8, 422. https://doi.org/10.3389/fpsyg.2017.00422.

Panadero, E., Klug, J., \& Järvelä, S. (2016). Third wave of measurement in the self-regulated learning field: when measuring and intervening come hand in hand. Scandinavian Journal of Educational Research, 60(6), 723-735. https://doi.org/10.1080/00313831.2015.1066436.

Pintrich, P. R., \& Garcia, T. (1994). Self-regulated learning in college students: Knowledge, strategies, and motivation. In P. R. Pintrich, D. R. Brown \& C.E. Weinstein (Hrsg.), Student motivation, cognition, and learning: essays in honor of Wilbert J. McKeachie (S. 113-133). Hillsdale: Lawrence Erlbaum.

Pintrich, P.R., \& Schunk, D.H. (2002). Motivation in education: theory, research, and applications (2. Aufl.). Upper Saddle River: Prentice Hall. 
Ragosta, P. (2010). The effectiveness of intervention programs to help college students acquire self-regulated learning strategies: A meta-analysis. Unpublished doctoral dissertation. New York: The City University of New York.

Rheinberg, F., Vollmeyer, R., \& Rollett, W. (2000). Motivation and action in self-regulated learning. In M. Boekaerts, P. R. Pintrich \& M. H. Zeidner (Hrsg.), Handbook of self-regulation (S. 503-529). San Diego: Academic Press.

Richardson, M., Abraham, C., \& Bond, R. (2012). Psychological correlates of university students' academic performance: a systematic review and meta-analysis. Psychological Bulletin, 138(2), 353-387. https://doi.org/10.1037/a0026838.

Rosário, P., Núñez, J. C., González-Pienda, J., Valle, A., Trigo, L., \& Guimarães, C. (2010). Enhancing selfregulation and approaches to learning in first-year college students: A narrative-based programme assessed in the Iberian Peninsula. European Journal of Psychology of Education, 25(4), 411-428. https://doi.org/10.1007/s10212-010-0020-y.

Questback GmbH (2016). Unipark (EFS Fall 2016). Oslo: Questback GmbH.

Sageder, J. (1994). Lernmotivation, Attributionstendenzen und Lernmethoden von Studienanfängern. Psychologie in Erziehung und Unterricht, 41, 120-133.

Schmidt, A. M., \& Ford, J. K. (2003). Learning within a learner control training environment: the interactive effects of goal orientation and metacognitive instruction on learning outcomes. Personnel Psychology, 56(2), 405-429. https://doi.org/10.1111/j.1744-6570.2003.tb00156.x.

Schmitz, B. (2001). Self-Monitoring zur Unterstützung des Transfers einer Schulung in Selbstregulation für Studierende. Zeitschrift für Pädagogische Psychologie, 15(3/4), 181-197. https://doi.org/10. 1024//1010-0652.15.34.181.

Schmitz, B., \& Wiese, B.S. (2006). New perspectives for the evaluation of intervention sessions in selfregulated learning: time-series analyses of diary data. Contemporary educational psychology, 31(1), 64-96. https://doi.org/10.1016/j.cedpsych.2005.02.002.

Schmitz, B., Klug, J., \& Schmidt, M. (2011). Assessing self-regulated learning using diary measures with university students. In B. J. Zimmerman \& D. H. Schunk (Hrsg.), Educational psychology handbook series. Handbook of self-regulation of learning and performance (S. 251-266). New York: Routledge/ Taylor \& Francis.

Schraw, G., Crippen, K. J., \& Hartley, K. (2006). Promoting Self-Regulation in Science Education: Metacognition as Part of a Broader Perspective on Learning. Research in Science Education, 36(1-2), 111-139. https://doi.org/10.1007/s11165-005-3917-8.

Schunk, D., \& Ertmer, P.A. (2000). Self-regulation and academic learning: self-efficacy enhancing interventions. In M. Boekaerts, P.R. Pintrich \& M. Zeidner (Hrsg.), Handbook of self-regulation (S. 631-649). San Diego: Academic Press.

Snow, R.E., Corno, L., \& Jackson, D. (1996). Conative and affective functions in educational psychology. In Handbook of Educational Psychology (S. 243-310). New York: Macmillan.

Spörer, N., \& Brunstein, J.C. (2006). Erfassung selbstregulierten Lernens mit Selbstberichtsverfahren: Ein Überblick zum Stand der Forschung. Zeitschrift für pädagogische Psychologie, 20(3), 147-160. https://doi.org/10.1024/1010-0652.20.3.147.

Van der Stel, M., \& Veenman, M. (2014). Metacognitive skills and intellectual ability of young adolescents: a longitudinal study from a developmental perspective. European Journal of Psychology of Education, 29(1), 117-137. https://doi.org/10.1007/s10212-013-0190-5.

Sung, Y.-T., Yang, J.-M., \& Lee, H.-Y. (2017). The effects of mobile-computer-supported collaborative learning: meta-analysis and critical synthesis. Review of educational research, 87(4), 768-805. https://doi.org/10.3102/0034654317704307.

Veenman, M. V.J., Van Hout-Wolters, B.H. A. M., \& Afflerbach, P. (2006). Metacognition and learning: conceptual and methodological considerations. Metacognition and Learning, 1(1), 3-14. https://doi. org/10.1007/s11409-006-6893-0.

Wagner, P., Spiel, C., \& Schober, B. (2006). Zeitmanagement. In H. Mandl \& H.F. Friedrich (Hrsg.), Handbuch Lernstrategien (S. 297-306). Göttingen: Hogrefe.

Weiner, B. (1985). An attributional theory of achievement motivation and emotion. Psychological review, 92(4), 548. https://doi.org/10.1037/0033-295X.92.4.548.

Wigfield, A., \& Eccles, J.S. (2000). Expectancy-value theory of achievement motivation. Contemporary Educational Psychology, 25(1), 68-81. https://doi.org/10.1006/ceps.1999.1015.

Winne, P. H. (1995). Inherent details in self-regulated learning. Educational Psychologist, 30(4), $173-187$. https://doi.org/10.1207/s15326985ep3004_2.

Wirth, J., \& Leutner, D. (2008). Self-regulated learning as a competence. Zeitschrift für Psychologie, 216(2), 102-110. https://doi.org/10.1027/0044-3409.216.2.102. 
Zimmerman, B.J. (2000). Attaining self-regulated learning: a social-cognitive perspective. In M. Boekaerts, P. Pintrich \& M. Zeidner (Hrsg.), Handbook of Self-regulation (S. 13-39). San Diego: Academic Press.

Zimmerman, B.J., \& Schunk, D.H. (2008). Motivation: An essential dimension of self-regulated learning. In D. H. Schunk \& B. J. Zimmerman (Hrsg.), Motivation and self-regulated learning: Theory, research, and applications (S. 1-30). New York: Routledge.

Zydney, J.M., \& Warner, Z. (2016). Mobile apps for science learning: Review of research. Computers \& Education, 94, 1-17. https://doi.org/10.1016/j.compedu.2015.11.001. 